\title{
Persuading communicating voters
}

Citation for published version (APA):

Kerman, T., \& Tenev, A. P. (2021). Persuading communicating voters. Maastricht University, Graduate School of Business and Economics. GSBE Research Memoranda No. 003 https://doi.org/10.26481/umagsb.2021003

Document status and date:

Published: 01/01/2021

DOI:

10.26481/umagsb.2021003

Document Version:

Publisher's PDF, also known as Version of record

\section{Please check the document version of this publication:}

- A submitted manuscript is the version of the article upon submission and before peer-review. There can be important differences between the submitted version and the official published version of record.

People interested in the research are advised to contact the author for the final version of the publication, or visit the DOI to the publisher's website.

- The final author version and the galley proof are versions of the publication after peer review.

- The final published version features the final layout of the paper including the volume, issue and page numbers.

Link to publication

\footnotetext{
General rights rights.

- You may freely distribute the URL identifying the publication in the public portal. please follow below link for the End User Agreement:

www.umlib.nl/taverne-license

Take down policy

If you believe that this document breaches copyright please contact us at:

repository@maastrichtuniversity.nl

providing details and we will investigate your claim.
}

Copyright and moral rights for the publications made accessible in the public portal are retained by the authors and/or other copyright owners and it is a condition of accessing publications that users recognise and abide by the legal requirements associated with these

- Users may download and print one copy of any publication from the public portal for the purpose of private study or research.

- You may not further distribute the material or use it for any profit-making activity or commercial gain

If the publication is distributed under the terms of Article $25 \mathrm{fa}$ of the Dutch Copyright Act, indicated by the "Taverne" license above, 


\section{Maastricht University}

Toygar Kerman, Anastas P. Tenev

Persuading Communicating Voters

$\mathrm{RM} / 21 / 003$

ISSN: $2666-8807$

\section{GSBE}

Maastricht University School of Business and Economics

Graduate School of Business and Economics

\section{P.O Box 616}

NL- 6200 MD Maastricht

The Netherlands 


\title{
Persuading Communicating Voters
}

\author{
By Toygar Kerman and Anastas P. Tenev*
}

January 5, 2021

\begin{abstract}
This paper studies a multiple-receiver Bayesian persuasion model, where a sender communicates with receivers who have homogeneous beliefs and aligned preferences. The sender wants to implement a proposal and commits to a communication strategy which sends private (possibly) correlated messages to the receivers, who are in an exogenous and commonly known network. Receivers can observe their neighbors' private messages and after updating their beliefs, vote sincerely on the proposal. We examine how networks of shared information affect the sender's gain from persuasion and find that in many cases it is not restricted by the additional information provided by the receivers' neighborhoods. Perhaps surprisingly, the sender's gain from persuasion is not monotonically decreasing with the density of the network.
\end{abstract}

Keywords: Bayesian Persuasion, Networks, Voting

JEL Classification: C72, D72, D82, D85

\section{Introduction}

Multiple-receiver Bayesian persuasion models with private communication often assume that receivers do not exchange information with each other between receiving signals from the sender and taking their action. In reality, however, people usually deliberate before voting in favor of a political party or simply before buying a product, and might consult friends and acquaintances in search of additional opinions and information. We model such communication among receivers prior to making a decision with a simple setup: we assume that receivers are in a fixed network that is commonly known and that neighbors can

*Department of Microeconomics and Public Economics (MPE), Maastricht University, The Netherlands (Kerman: t.kerman@maastrichtuniversity.nl; Tenev: ap.tenev@maastrichtuniversity.nl). We would like to thank Jean-Jacques Herings, Dominik Karos, Ronald Peeters and Frank Thuijsman for their valuable comments and suggestions. We would also like to thank the participants of ASSET 2020 Virtual Meeting, 15th BiGSEM Doctoral Workshop on Economics and Management, GamesNet Webinar Series, and Maastricht University MLSE Seminar Series. 
observe each other's private messages. An application of such communication are social networks like Facebook, where parties can target political adverts at specific (potential) voter groups and such use of social media has become a common feature of election campaigns in recent years. In this setting, if a person likes or shares an ad or a video, it is visible to all of their friends on the social network. In this paper we consider the limit case, where the sender sends perfectly custom-tailored messages to the receivers and the messages are observed by the receivers and their direct neighbors with probability one.

This setup significantly complicates the sender's problem of optimal persuasion as he must also take into account the intricacies of the information flow between receivers when deciding how to design his communication strategy. In the absence of a network structure, the sender can improve upon public communication by employing private messages. Hence, the immediate question arises whether private communication is still (strictly) beneficial for the sender when receivers communicate within a network. If so, are some networks more manipulable than others? Is the most manipulable network empty? Can the sender to benefit from a denser network?

\subsection{An Illustrative Example}

Suppose that a company, which is either profitable $(\mathrm{P})$ or not profitable $(\mathrm{N})$, has two potential investors, $I_{1}$ and $I_{2}$, who initially believe it to be profitable with probability $1 / 3$. Investors will invest in the company only if they consider it is profitable with probability at least $1 / 2$. Both parties can potentially give the same amount, and the investment from only one of them is sufficient to cover the company's cash flow problems. Two financial reports are prepared regarding the company's finances, where each report is randomly assigned (with equal probability) to one of the investors. While one report provides true findings, the other one always favors investment.

First, assume that investors are not communicating, i.e. they are in the empty network. The communication strategy of the CEO can be formalized by distributions $\pi(\cdot \mid P)$ and $\pi(\cdot \mid N)$ on some set of signals. Let $(p, n)$ denote the signal in which $I_{1}$ receives a message to invest and $I_{2}$ a message not to invest. The CEO's communication strategy above can be represented as follows.

\begin{tabular}{c|cc}
$\pi$ & $P$ & $N$ \\
\hline$(p, p)$ & 1 & 0 \\
$(p, n)$ & 0 & $\frac{1}{2}$ \\
$(n, p)$ & 0 & $\frac{1}{2}$
\end{tabular}

After observing $p$, any investor's belief that the company is profitable is $1 / 2$. Hence, after any realization at least one of them invests. The CEO guarantees at least one investment with probability 1 and the communication strategy above is indeed optimal.

Now, assume that $I_{1}$ and $I_{2}$ know each other and exchange the information they got from the reports before making their decisions. This can be presented with the network: 


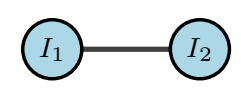

Since investors can observe each other's messages, the communication strategy above is no longer optimal: when the signal realization is $(p, n)$ or $(n, p)$, both investors know that the company is not profitable, i.e. the true state is $N$. In this case, the probability of obtaining an investment is determined by the probability of $(p, p)$, i.e. $1 / 3$. Since receivers can observe the signal realization (as the network is complete), the CEO cannot communicate privately and alternatively chooses the following communication strategy:

\begin{tabular}{c|cc}
$\pi^{\prime}$ & $P$ & $N$ \\
\hline$(p, p)$ & 1 & $\frac{1}{2}$ \\
$(n, n)$ & 0 & $\frac{1}{2}$
\end{tabular}

Note that since the communication strategy is public, either both investors invest or none of them does. The probability of obtaining an investment under $\pi^{\prime}$ is $1 / 3 \cdot 1+2 / 3 \cdot 1 / 2=$ $2 / 3$. Since it is sufficient for the CEO to guarantee only one investment, the CEO is worse off when the investors are communicating.

The example illustrates how the additional exchange of information affects the Sender's gain from persuasion in two extreme cases: the empty network when there is no link between $I_{1}$ and $I_{2}$, and the complete network in the latter case. In a complete network, it is clear that the sender's optimal communication strategy is public. This paper shows that in some networks that are neither empty nor complete (and for some voting quotas) the sender's optimal communication strategy is indeed public. However, in others he can still benefit from communicating privately, or even achieve the optimal probability of success as under the empty network.

\subsection{Overview of Results}

We consider an exogenous network that is common knowledge, a binary state space, and a sender who commits to a private communication strategy. Receivers know the joint distribution of signals (vectors of messages), but only observe their own and their neighbors' private messages from the signal realization. If the network is empty, then our model reduces to the model of Kerman, Herings, and Karos (2020), which is used as a benchmark.

First, we show that for non-empty networks the "traditional" assumptions for multiplereceiver Bayesian persuasion models are no longer without loss of generality. The most common of these assumptions, straightforwardness, under which the sender sends "recommendations" to vote for an alternative and receivers follow the recommendations, does not hold since receivers take into account not only their own messages, but also their neighbors'. The shared information limits the possible message combinations the sender can use for optimal persuasion as the recommendations are no longer fully private. 
Another such assumption that is not without loss of generality is "truth-telling" in the sender's preferred state. In other words, it is not always in the sender's best interest to generate a perfectly informative message whenever the true state is his preferred one; there exist networks (e.g. the line network) where the sender is better off garbling information in such cases. This is due to the fact that, in addition to different messages, the variability between the signals in the sender's preferred state is used to limit the information flow between neighbours.

Given a number of receivers and a quota, the sender cannot achieve a higher probability of success in a non-empty network than under the empty network of the same size. The reason for this is that given any non-empty network and any communication strategy $\pi$, there exists a communication strategy $\pi^{\prime}$ which reveals exactly the same information in the empty network as $\pi$ does in the non-empty network. In other words, any communication strategy in a non-empty network can be replicated in its corresponding empty network. In contrast, when the network is complete, the optimal communication strategy is public, as illustrated in the motivating example. For networks that lie in between the empty network and the complete network, we consider network structures which are often discussed in the literature (star, wheel, circle and other regular networks) and identify conditions under which the sender can achieve the probability of success under the empty network. Interestingly, if there are sufficiently many isolated nodes in a network, this can be achieved regardless of how many or between which of the remaining receivers the links are.

Finally, perhaps surprisingly, while it seems intuitive that adding a link to a given network would (weakly) decrease the optimal value (as receivers gain more information), it is possible that the optimal value is higher in a denser network. The reasoning behind this is that while any communication strategy in a non-empty network can be replicated in the empty network of the same size, it is not always the case that it can be replicated in a less dense network which is non-empty. Due to this fact, it is possible that an optimal communication strategy under a denser network has a higher value than an optimal communication strategy under a less dense network.

The rest of the paper is organized as follows. Subsection 1.3 discusses related literature. Section 2 provides notation and preliminary definitions. Section 3 discusses the benchmark case and shows preliminary results. Section 4 gives observations which hold for network structures in general. Section 5 focuses on the optimal values in specific networks. Section 6 shows that a denser network is not always worse for the sender. Section 7 concludes.

\subsection{Related Literature}

The current model comes closest to and is an extension of Kerman et al. (2020), which builds upon Kamenica and Gentzkow (2011) and considers a sender communicating privately with multiple receivers. The authors derive an optimal communication strategy under the assumption that receivers vote sincerely. While we also assume sincere voting, 
a crucial difference to the current setup is that in their model a receiver only has access to information revealed to them by the sender, whereas in our setup directly connected voters perfectly exchange information. So, their model is a special case of ours, applied to the empty network setup. While they focus on finding an optimal communication strategy that yields an equilibrium under sincere voting, we assume sincere voting and determine optimal communication strategies for different types of networks.

The current paper also relates to the literature on public communication and collective decision making. Schnakenberg (2015) considers an expert who is privately informed about the state and can conceal information from the receivers. One important distinction from our paper is that the expert does not have to commit to a communication strategy, which in effect reduces the ex ante expected utilities of the voters. Alonso and Câmara (2016) study public communication where receivers have heterogeneous preferences which the sender can exploit by targeting minimal winning coalitions. Kosterina (2018) considers a model with a continuous state space in which receiver's prior is unknown and shows that the solution to the problem is the same as the one of persuading receivers with heterogeneous priors. She proves that even when the receiver's prior is unknown, the optimal communication strategy always includes a signal that tells the truth with probability 1, whereas in our setup this is not generally true.

Our paper also relates to the literature on voting games and private communication. Wang (2013) compares the outcomes from public and private communication strategies but also incorporates strategic voter behavior in the analysis and concludes that the sender is weakly worse off under private communication when messages are uncorrelated. In contrast, we consider private correlated messages and show that this often improves upon public communication. Bardhi and Guo (2018) also focus on collective decision making and in particular the unanimity voting rule, whereas we allow for general monotonic and anonymous voting rules. Chan, Gupta, Li, and Wang (2019) consider private communication as well and assume that voting is costly. In their model the optimal communication strategy targets the receivers with the lowest costs who are easiest to persuade.

This paper is also related to the literature on more general games in information design. Bergemann and Morris (2016) consider a game of incomplete information, characterize Bayesian Nash equilibria and demonstrate that this corresponds to Bayes correlated equilibria. They show that in equilibrium receivers are "obedient", they follow the recommendation by the sender. Taneva (2019) derives the optimal information structure in finite environments and characterizes it in a symmetric binary setting. She shows that a related notion dubbed "directness" (which also corresponds to straightforwardness in the sense of Kamenica and Gentzkow (2011)) can be used without loss of generality. One important difference of our paper to these is that we do not consider strategic receivers. Moreover, our setting does not allow for straightforwardness.

One paper that considers Bayesian persuasion in networks is Egorov and Sonin (2019). In their model, a sender communicates publicly with the receivers who are in a fixed network by choosing the level of propaganda. A receiver might choose to get the information 
from the signal of the sender for a cost, or rely on his neighbors and get to the same information from them. The authors find that adding or removing links from the network can have different effects on the level or propaganda, depending on whether the probability that information passes through two agents is high or low. Unlike their paper, in our model the sender does not give identical information to receivers, but rather sends private messages to each receiver which are observed by his neighbors at no cost.

Our paper also shares some characteristics with Buechel and Mechtenberg (2019), where agents are in a network and experts can share with their direct neighbors a policy recommendation on the basis of private signals they receive. The authors find that the outcomes crucially depend on the network structure, and more precisely on whether there are experts with disproportionally large neighborhoods. In this case, it might be beneficial for their neighbors (the non-experts) to ignore such recommendations. While their main model includes only bipartite networks, the authors extend it to general network structures and analyse equilibrium strategies. Unlike our setup, they have strategic receivers and agents decide whether or not to share information with their neighborhood, e.g. they can ignore a connection with their neighbor.

Candogan and Drakopoulos (2020) consider a model of social network interactions in which agents choose whether or not to engage with some (possibly inaccurate) content on the social network and the agents' payoffs depend on the engagement of their neighbors. The platform tries to design a signalling mechanism which maximizes engagement or minimizes misinformation by sending recommendations to its users to engage or not. They find that in order to maximize engagement the platform needs to issue a recommendation to engage based on an agent-specific threshold determined their position in the network, while to minimize misinformation common thresholds are used. In another model with local strategic complementarities Candogan (2019) finds that when the degrees of some nodes in the network increase, this can reduce the information designer's payoff.

\section{Notation}

\subsection{Communication Strategy}

Let $N=\{1, \ldots, n\}$ be the set of receivers and $\Omega=\{X, Y\}$ the set of states of the world. Sender and receivers share a common prior belief $\lambda^{0} \in \Delta^{o}(\Omega)$ about the true state of the world, where $\Delta^{o}(\Omega)$ denotes the set of strictly positive probability distributions on $\Omega$.

Let $S_{i}$ be a finite set of messages the sender can send to receiver $i$, and let $S=\prod_{i \in N} S_{i}$, where the elements of $S$ are called signals. A communication strategy is a function $\pi$ : $\Omega \rightarrow \Delta(S)$ which maps each state of the world to a joint probability distribution over signal realizations. Denote the set of all communication strategies by $\Pi$.

For each signal $s \in S$, let $s_{i} \in S_{i}$ denote the message for receiver $i$. For each $s_{i} \in S_{i}$ and

$\omega \in \Omega$, let $\pi_{i}\left(s_{i} \mid \omega\right)=\sum_{t \in S: t_{i}=s_{i}} \pi(t \mid \omega)$, which is the probability that receiver $i$ observes $s_{i}$ given $\omega$. Define $S^{\pi}=\{s \in S \mid \exists \omega \in \Omega: \pi(s \mid \omega)>0\}$. That is, $S^{\pi}$ consists of signals in 
$S$ which are sent with positive probability by $\pi$. Similarly, for each $\pi$ and $i \in N$, define $S_{i}^{\pi}=\left\{s_{i} \in S_{i} \mid \exists \omega \in \Omega: \pi_{i}\left(s_{i} \mid \omega\right)>0\right\}$, which is the set of messages receiver $i$ observes with positive probability under $\pi$.

\subsection{Networks}

An undirected network is a map $g: N \times N \rightarrow\{0,1\}$ with $g_{i j}=g(i, j)$ and $g_{i j}=g_{j i}$. Given a set of receivers $N$, let $G(N)$ be the set of all such networks. We assume that receivers are in a fixed network, which is common knowledge among the sender and receivers. Each receiver in the network can observe his neighbors' message realizations. Thus, in a nonempty network a receiver gathers more information about the true state than he would from the same communication strategy under the empty network.

A network $g \in G(N)$ is complete if for all $i, j \in N$ with $i \neq j$ it holds that $g_{i j}=1$, i.e. every two nodes have a link. In this case each receiver knows the signal realization, so all communication strategies are public on the complete network. For any network $g \in G(N)$, we denote the empty network with the same number of receivers by $g_{0}$. Observe that an empty network corresponds to a standard multiple-receiver Bayesian persuasion model.

Let $N_{i}(g)=\left\{j \in N \mid g_{i j}=1\right\}$ be the neighborhood of receiver $i$ in $g$ and let $\delta_{i}^{g}=\left|N_{i}(g)\right|$ denote the degree of $i$ in $g$. Let $\bar{N}_{i}(g)=N_{i}(g) \cup\{i\}$. For any $\pi \in \Pi, s \in S^{\pi}, i \in N$, and $j \in N_{i}(g)$, let $s_{i j}$ be the message $i$ observes from $j$ in $s$, that is $s_{i j}=s_{j}$. Let $s_{i}(g)=\left(s_{i j}\right)_{j \in \bar{N}_{i}(g)}$ be the information neigborhood of receiver $i$ in $s$, that is, $s_{i}(g)$ is the vector of messages (with length $\delta_{i}^{g}+1$ ) receiver $i$ observes upon signal realization $s$. Let $A_{i}^{\pi}(g, s)=\left\{t \in S^{\pi} \mid t_{i}(g)=s_{i}(g)\right\}$ be the set of signals $i$ considers possible upon signal realization $s$, or in other words, the set of signals $i$ associates with $s$. Given $s, t \in S^{\pi}$, we say that $t$ is associated with $s$ if there exists an agent $i \in N$ such that $t \in A_{i}^{\pi}(g, s)$. Let $A^{\pi}(g, s)=\cup_{i \in N} A_{i}^{\pi}(g, s)$ be the set of all signals associated with $s$.

For any $g \in G(N), \pi \in \Pi$, and $s \in S^{\pi}$, the posterior belief vector $\lambda^{s, g} \in \Delta(\Omega)^{n}$ is defined by:

$$
\lambda_{i}^{s, g}(\omega)=\frac{\sum_{t \in A_{i}^{\pi}(g, s)} \pi(t \mid \omega) \lambda^{0}(\omega)}{\sum_{\omega^{\prime} \in \Omega} \sum_{t \in A_{i}^{\pi}(g, s)} \pi\left(t \mid \omega^{\prime}\right) \lambda^{0}\left(\omega^{\prime}\right)}, \quad i \in N, \omega \in \Omega .
$$

That is, $\lambda_{i}^{s, g}(\omega)$ is receiver $i$ 's posterior belief that the state is $\omega$ upon observing $s_{i}(g)$.

A communication strategy $\pi \in \Pi$ induces $\sigma^{g} \in \Delta\left(\Delta(\Omega)^{n}\right)$ under network $g$ if for all $\lambda \in \Delta(\Omega)^{n}$ it holds that:

$$
\sigma^{g}(\lambda)=\sum_{s \in S^{\pi}: \lambda^{s, g}=\lambda} \sum_{\omega \in \Omega} \pi(s \mid \omega) \lambda^{0}(\omega) .
$$

In words, $\sigma^{g}(\lambda)$ is the probability of posterior vector $\lambda$ under network $g .{ }^{1}$

\footnotetext{
${ }^{1}$ If $\lambda \notin \operatorname{supp}\left(\sigma^{g}\right)$, then the right hand side of (2) is 0. Moreover, it is well-defined since $S^{\pi}$ is finite.
} 


\section{$2.3 \quad$ Voting}

For each $i \in N$, let $B_{i}=\{x, y\}$ be the set of actions of receiver $i$. Let $B=\prod_{i \in N} B_{i}$ denote the space of action profiles and let $Z=\{x, y\}$ be the set of voting outcomes. Following the signal realization, each receiver chooses an action according to his posterior belief.

Let $z^{k}: B \rightarrow Z$ be a map, where $z^{k}(a)$ is the outcome of the vote when the action profile is $a$ and is defined by:

$$
z^{k}(a)= \begin{cases}x & \text { if }\left|\left\{i \in N: a_{i}=x\right\}\right| \geq k, \\ y & \text { otherwise. }\end{cases}
$$

We assume that the sender's utility function $v: Z \rightarrow\{0,1\}$ has value 1 if $x$ is implemented and 0 otherwise.

For any $g \in G(N), \pi \in \Pi$, and $i \in N$, let $S_{i}^{\pi}(g)=\prod_{j \in \bar{N}_{i}(g)} S_{j}^{\pi}$ be the space of vectors of length $\delta_{i}^{g}+1$ that $i$ can observe under $g$ and $\pi$. Let $\alpha_{i}^{\pi, g}: S_{i}^{\pi}(g) \rightarrow B_{i}$ be agent $i$ 's sincere action function, such that for any realization $s \in S^{\pi}$ it holds that:

$$
\alpha_{i}^{\pi, g}\left(s_{i}(g)\right)= \begin{cases}x & \text { if } \lambda_{i}^{s, g}(X) \geq \frac{1}{2}, \\ y & \text { otherwise }\end{cases}
$$

Throughout the paper we assume that $\lambda^{0}(X)<\lambda^{0}(Y)$, since otherwise there is no need for persuasion. Define $Z_{x}^{g}(\pi)=\left\{s \in S^{\pi} \mid z^{k}\left(\alpha^{\pi, g}(s)\right)=x\right\}$. That is, $Z_{x}^{g}(\pi)$ is the set of signals which implement $x$ in $g$ under $\pi$ and sincere voting.

Receiver $i$ is pivotal in $s \in S^{\pi}$ if for any $a_{i} \in B_{i}, z^{k}\left(a_{i}, \alpha_{-i}^{\pi, g}\left(s_{-i}(g)\right)=a_{i}\right.$. That is, $i$ is pivotal in the voting following $s$ if $i$ 's vote determines the voting outcome given that all $j \neq i$ vote sincerely.

Let $a \in B$ be an action profile and $z=z^{k}(a)$ be a voting outcome. The value of a communication strategy $\pi \in \Pi$ for quota $k$ is defined as the sender's expected utility under distribution $\sigma^{g}$ induced by $\pi$ in network $g$. As we fix $\lambda^{0}$ and $\alpha_{i}^{\pi, g}$ throughout the paper, we write $V_{k}^{\pi}(g)=V_{k}^{\pi}\left(\lambda^{0}, g, \alpha^{\pi, g}\right)$. The value of the sender is:

$$
V_{k}^{\pi}(g)=\mathbb{E}_{\lambda^{0}}\left[\mathbb{E}_{\pi}\left[v\left(z^{k}\left(\alpha^{\pi, g}(s)\right)\right]\right]=\lambda^{0}(X) \sum_{s \in Z_{x}^{g}(\pi)} \pi(s \mid X)+\lambda^{0}(Y) \sum_{s \in Z_{x}^{g}(\pi)} \pi(s \mid Y) .\right.
$$

Thus, the value of a communication strategy is equal to the probability of $x$ being implemented under $\pi$ and $g$ and quota $k$, given that receivers vote sincerely.

A communication strategy $\pi^{*}$ is optimal in $\Pi$ under $g$ for quota $k$ if $V_{k}^{\pi^{*}}(g)=$ $\sup _{\pi \in \Pi} V_{k}^{\pi}(g)$. The value of an optimal communication strategy on the empty network with $n$ nodes and quota $k$ is denoted by $V_{k}^{n}$.

\section{Preliminaries}

This section demonstrates how the information-sharing feature of our model produces a non-trivial change in the overall setup of multiple-receiver Bayesian persuasion as many 
of the common assumptions in standard models cease to hold in general. First, we briefly consider the model in Kerman et al. (2020), which corresponds to the empty network case in our framework and is used as a benchmark. Second, we demonstrate that the network structure can affect the agents' posteriors and the (optimal) value. Third, we show that the notion of straightforwardness as defined in Kamenica and Gentzkow (2011) does not hold for optimal strategies in our model. Finally, sending a recommendation with probability 1 in the sender's preferred state, an assumption that is made without loss of generality for optimal strategies in many Bayesian persuasion models, is also no longer optimal.

\subsection{Optimal Communication Strategy on the Empty Network and with Public Signals}

In Kerman et al. (2020), it is without loss of generality to restrict attention to straightforward (in the sense of Kamenica and Gentzkow (2011)) and anonymous communication strategies. Given a binary state space $\Omega=\{X, Y\}$, it is optimal for the sender to send "recommendations", $x$ or $y$, as messages. In this case, the probability that $x$ is sent to exactly $\ell$ receivers if the state is $X$ and the probability that $x$ is sent to exactly $\ell$ receivers if the state is $Y$ can be represented by $q_{\ell}$ and $r_{\ell}$, respectively, where each signal in which the same number of receivers observe $x$ has the same probability. An optimal communication strategy (in the empty network) is then given by the following theorem.

Theorem 3.1. (Kerman et al., 2020) Let $\pi^{*} \in \Pi$ with representation $\left(q^{*}, r^{*}\right)$ be:

$$
\left(q_{n}^{*} ; r_{0}^{*}, r_{k}^{*}\right)= \begin{cases}(1 ; 0,1) & \text { if } \lambda^{0}(X) \geq \frac{k}{n+k}, \\ \left(1 ; 1-\frac{\lambda^{0}(X)}{\lambda^{0}(Y)} \frac{n}{k}, \frac{\lambda^{0}(X)}{\lambda^{0}(Y)} \frac{n}{k}\right) & \text { if } \lambda^{0}(X)<\frac{k}{n+k} .\end{cases}
$$

Then $\pi^{*}$ is optimal at $\lambda^{0}$. In particular, $V_{k}^{n}=\min \left\{\frac{n+k}{k} \lambda^{0}(X), 1\right\}$.

Note that the optimal public communication strategy's value is always independent of the network and the quota since all agents observe the signal realization. In this case, since receivers have homogenous prior beliefs, the situation is the same as persuading a single receiver. Thus, as in Kamenica and Gentzkow (2011), $x$ is implemented with probability 1 in state $X$ and with probability $\lambda^{0}(X) / \lambda^{0}(Y)$ in state $Y$. The value of the optimal public communication strategy is then given by $V^{p}=\lambda^{0}(X) \cdot 1+\lambda^{0}(Y) \cdot \lambda^{0}(X) / \lambda^{0}(Y)=2 \lambda^{0}(X)$.

\subsection{The Effects of the Network}

One important observation in our framework is that the set of posteriors which can be induced differs from the one under a model without a network structure. Given a communication strategy $\pi \in \Pi, s \in S^{\pi}$ and $g, g^{\prime} \in G(N)$, let $\lambda^{s, g}$ and $\lambda^{s, g^{\prime}}$ be the posterior vectors under $g$ and $g^{\prime}$, respectively. It is easy to see that for $g \neq g^{\prime}$, it is possible that 
$\lambda^{s, g} \neq \lambda^{s, g^{\prime}}$. If $g \subseteq g^{\prime}$, an agent gathers more information upon a signal realization under $g^{\prime}$ than under $g$ by also observing the messages of his new neighbors, which leads to different posterior beliefs in the two models. This is shown in Example 3.2.

Example 3.2. Let $|N|=3, \lambda^{0}(X)=1 / 3$, and $k=2$. The optimal communication strategy $\pi$ prescribed by Theorem 3.1 for the empty network $g_{0}$ is given by $q_{3}^{*}=1$, $r_{2}^{*}=3 / 4$, and $r_{0}^{*}=1 / 4$, since $\lambda^{0}(X)<k /(n+k)$. This can be represented by:

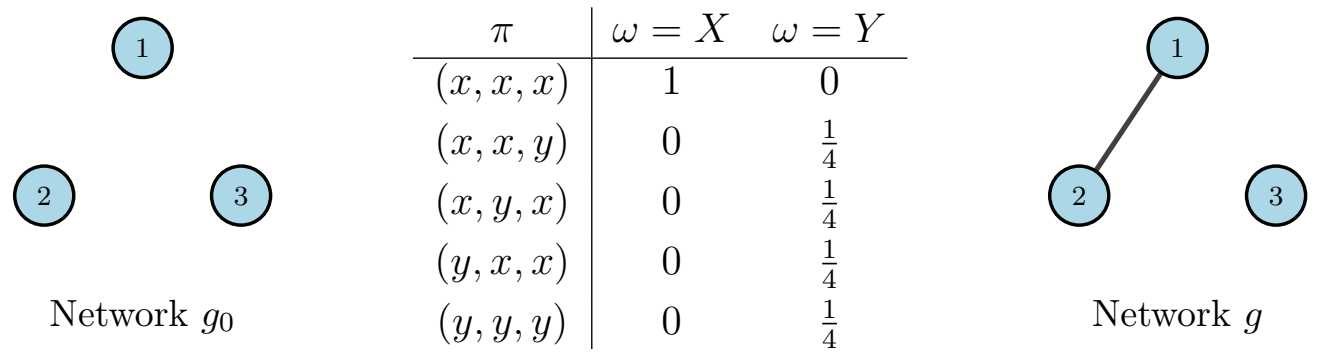

The support of the distribution that $\pi$ induces under the empty network $g_{0}$ is given by:

$$
\operatorname{supp}\left(\sigma^{g_{0}}\right)=\left\{\left(\frac{1}{2}, \frac{1}{2}, \frac{1}{2}\right),\left(\frac{1}{2}, \frac{1}{2}, 0\right),\left(\frac{1}{2}, 0, \frac{1}{2}\right),\left(0, \frac{1}{2}, \frac{1}{2}\right),(0,0,0)\right\},
$$

and the value is $V_{2}^{3}=V_{2}^{\pi}\left(g_{0}\right)=5 / 6$.

Now consider network $g$ on the right-hand side, which has one added link compared to its counterpart $g_{0}$, so that receivers 1 and 2 can observe each other's messages. The set of posteriors induced by $\pi$ under $g$ is:

$$
\operatorname{supp}\left(\sigma^{g}\right)=\left\{\left(\frac{2}{3}, \frac{2}{3}, \frac{1}{2}\right),\left(\frac{2}{3}, \frac{2}{3}, 0\right),\left(0,0, \frac{1}{2}\right),(0,0,0)\right\} .
$$

The value is $V_{2}^{\pi}(g)=1 / 2<5 / 6=V_{2}^{\pi}\left(g_{0}\right)$. There are fewer posterior vectors under $g$ than under the empty network $g_{0}$. Since receivers 1 and 2 can observe each other's messages, different signals lead to the same posterior vector. Note that 1 and 2 would have the same posterior belief in any communication strategy. Since they have the same information neighborhood, they always update their beliefs in the same way. It follows that the sender can send the same message to 1 and 2 without loss of generality. ${ }^{2}$ Thus, since $k=2$ the problem is equivalent to persuading either receivers 1 and 2 together, or persuading all receivers. However, this is tantamount to employing a public communication strategy in which case the network does not have an effect since all agents observe the same message. Therefore, the optimal communication strategy in $g$ is public and given by:

\begin{tabular}{c|cc}
$\pi^{\prime}$ & $\omega=X$ & $\omega=Y$ \\
\hline$(x, x, x)$ & 1 & $\frac{1}{2}$ \\
$(y, y, y)$ & 0 & $\frac{1}{2}$
\end{tabular}

Thus, the value is $V^{\pi^{\prime}}(g)=1 / 3 \cdot 1+2 / 3 \cdot 1 / 2=2 / 3=V^{p}$. So, if $|N|=3$ and $k=2$, having even one link between agents reduces the optimal value from the one under the empty network, $V_{2}^{3}$, to the value of the optimal public communication strategy, $V^{p}$.

\footnotetext{
${ }^{2} \mathrm{~A}$ generalized version of this statement is proved later on in Proposition 4.4.
} 
An intuitive observation in Example 3.2 is that adding a link to the network while keeping $\pi$ (which is optimal on $g_{0}$ ) fixed decreases the value of $\pi$. However, it is also possible that the sender benefits from a denser network under a fixed $\pi$. We show this next.

Example 3.3. Let $|N|=5, \lambda^{0}(X)=1 / 3$ and $k=3$. Consider the following network $g$ and communication strategy $\pi$ :

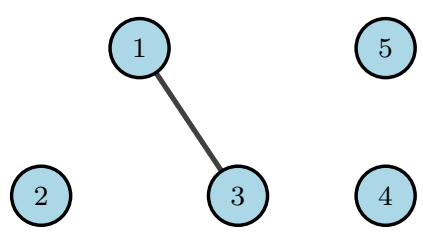

\begin{tabular}{c|cc}
$\pi$ & $\omega=X$ & $\omega=Y$ \\
\hline$(x, y, x, x, y)$ & $\frac{1}{3}$ & $\frac{1}{6}$ \\
$(x, y, x, y, x)$ & $\frac{1}{3}$ & $\frac{1}{6}$ \\
$(y, y, y, x, x)$ & $\frac{1}{6}$ & $\frac{1}{6}$ \\
$(y, y, y, y, y)$ & $\frac{1}{6}$ & $\frac{1}{2}$
\end{tabular}

The support of $\sigma^{g}$ is given by:

$$
\operatorname{supp}\left(\sigma^{g}\right)=\left\{\left(\frac{1}{2}, \frac{1}{3}, \frac{1}{2}, \frac{3}{7}, \frac{3}{11}\right),\left(\frac{1}{2}, \frac{1}{3}, \frac{1}{2}, \frac{3}{11}, \frac{3}{7}\right),\left(\frac{1}{5}, \frac{1}{3}, \frac{1}{5}, \frac{3}{7}, \frac{3}{7}\right),\left(\frac{1}{5}, \frac{1}{3}, \frac{1}{5}, \frac{3}{11}, \frac{3}{11}\right)\right\} .
$$

Thus, $x$ is not implemented after any realization, i.e. $V_{3}^{\pi}(g)=0$.

Now, consider the following network $g^{\prime}$.

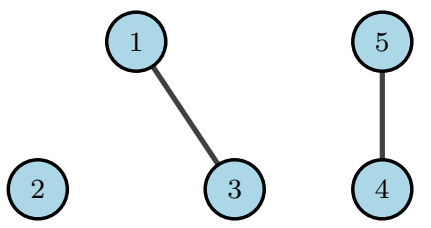

The beliefs of receivers 1, 2 and 3 are the same under $g^{\prime}$ as under $g$ but receivers 4 and 5 have different beliefs. The support under the same communication strategy $\pi$ is:

$$
\operatorname{supp}\left(\sigma^{g^{\prime}}\right)=\left\{\left(\frac{1}{2}, \frac{1}{3}, \frac{1}{2}, \frac{1}{2}, \frac{1}{2}\right),\left(\frac{1}{5}, \frac{1}{3}, \frac{1}{5}, \frac{1}{3}, \frac{1}{3}\right),\left(\frac{1}{5}, \frac{1}{3}, \frac{1}{5}, \frac{1}{7}, \frac{1}{7}\right)\right\} .
$$

Thus, $x$ is implemented after realizations $(x, y, x, x, y)$ and $(x, y, x, y, x)$ as both lead to the posterior vector $\left(\frac{1}{2}, \frac{1}{3}, \frac{1}{2}, \frac{1}{2}, \frac{1}{2}\right)$. Hence:

$$
V_{3}^{\pi}\left(g^{\prime}\right)=\frac{1}{3} \cdot \frac{2}{3}+\frac{2}{3} \cdot \frac{1}{3}=\frac{4}{9}>0=V_{3}^{\pi}(g),
$$

so, the sender benefits from the same communication strategy in a denser network.

While the sender benefits from the additional connection provided in $g^{\prime}$, a notable aspect of Example 3.3 is that $\pi$ is not optimal on $g$. This leaves the question how adding a link generally affects the value of the optimal strategy. In Example 3.2, the optimal communication strategy $\pi$ under $g_{0}$ has a strictly higher value than the optimal communication strategy $\pi^{\prime}$ under the denser network $g$. One might expect this to be a general observation. However, Section 6 shows that this is not always true. 


\subsection{Straightforwardness}

Translating the straightforwardness definition of Kamenica and Gentzkow (2011) to multiple receivers, a communication strategy $\pi \in \Pi$ is straightforward if $S_{i}^{\pi} \subseteq B_{i}$ and if for the sincere action function $\alpha_{i}^{\pi}: S_{i}^{\pi} \rightarrow B_{i}$ and $a_{i} \in S_{i}^{\pi}$ it holds that $\alpha_{i}^{\pi}\left(a_{i}\right)=a_{i}$. First, observe that under the sincere action function $\alpha_{i}^{\pi}$ receivers vote only according to their own message, whereas in our model the sincere action function $\alpha_{i}^{\pi, g}$ also takes into account their neighbors' messages. Hence, using the standard definition of straightforwardness is not without loss of generality. This observation is illustrated in Example 3.6 below. However, first we need to introduce a definition and a lemma.

Upon a signal realization, a receiver votes for $x$ if the probability of observing their specific message vector is sufficiently higher in state $X$ than in state $Y$, given their prior belief and a specified communication strategy. In order for this to hold, there must exist at least one signal which includes this message vector and has higher probability in state $X$. Such signals are instrumental for increasing the probability of implementing $x$ and will be referred to as "anchors", because the $x$ votes are dependent on them.

Definition 3.4. For any $\pi \in \Pi$ and $s \in S^{\pi}$, the signal $s$ is an anchor if $\pi(s \mid X) \lambda^{0}(X) \geq$ $\pi(s \mid Y) \lambda^{0}(Y)$. The set of all anchors is denoted by $A n(\pi)$.

Given $\pi \in \Pi, s \in A n(\pi)$ and $i \in N$, if for every $t \in S^{\pi}$ with $t \neq s$ it holds that $s_{i}(g) \neq t_{i}(g)$, then $\alpha_{i}^{\pi, g}\left(s_{i}(g)\right)=x$. That is, if a receiver $i$ can uniquely identify the signal realisation as an anchor, he votes for $x$.

Lemma 3.5. Let $g \in G(N)$. For any $\pi \in \Pi$ with $V_{k}^{\pi}(g)>0$ it holds that $\operatorname{An}(\pi) \neq \emptyset$.

The proofs to all statements can be found in the Appendix. Lemma 3.5 shows that every communication strategy with a non-zero value has at least one anchor. Its proof further implies that every $x$-vote in such a strategy is associated with at least one anchor.

Now we can show that assuming straightforwardness is not without loss of generality.

Example 3.6. Let $|N|=3, \lambda^{0}(X)=1 / 3$, and $k=1$. Consider the following network $g$ :

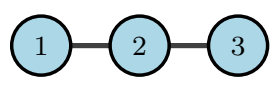

\begin{tabular}{c|cc}
$\pi^{\prime}$ & $\omega=X$ & $\omega=Y$ \\
\hline$(x, x, x)$ & 1 & 0 \\
$(y, x, x)$ & 0 & $\frac{1}{2}$ \\
$(x, x, y)$ & 0 & $\frac{1}{2}$
\end{tabular}

Let us try to construct a straightforward communication strategy $\pi$. First, note that $\lambda_{2}^{s, g}(X) \geq 1 / 2$ if and only if $s \in A n(\pi)$, so receiver 2 never votes for $x$ in any non-anchor signal. This is the case since for any $s \in S^{\pi}, s_{2}(g)=s$, that is, receiver 2 always observes the whole signal as he has access to the messages of everyone in the network. Therefore, for any straightforward communication strategy $\pi \in \Pi$ it must hold that: (i) $t_{2}=y$ for all $t \notin A n(\pi)$ and (ii) $s_{2}=x$ for all $s \in A n(\pi)$. However, since for any such $s$ and $t$ it 
holds that $s_{2} \neq t_{2}$, we have $\lambda_{1}^{t, g}(X), \lambda_{3}^{t, g}(X)<1 / 2$, as observing 2 would induce 1 and 3 to also vote $y$ in non-anchor signals. Thus, it must also hold that: (i) $t=(y, y, y)$ for all $t \notin A n(\pi)$ and (ii) $s_{2}=x$ for all $s \in A n(\pi)$. This implies that for any possible quota $k=1,2,3, V_{k}^{\pi}(g)=V^{p}=2 / 3$, so the maximum value of a straightforward communication strategy would be equal to the value of the optimal public communication strategy.

In contrast, the non-straightforward communication strategy $\pi^{\prime}$ above can improve on this value since:

$$
\operatorname{supp}\left(\sigma^{g}\right)=\left\{\left(\frac{1}{2}, 1, \frac{1}{2}\right),\left(0,0, \frac{1}{2}\right),\left(\frac{1}{2}, 0,0\right)\right\}
$$

and $V_{1}^{\pi}(g)=1$, which is clearly optimal as it is the maximal possible value. Here it is important to note that under $\pi^{\prime}$ after observing the same message $(x)$, receiver 2 votes for $x$ in some signals and for $y$ in others. ${ }^{3}$

A naive extension of staightforwardness to the network setting would suggest that strategies send recommendations to neighborhoods rather than individual receivers, where a receiver votes for $x$ if he observes only $x$ in his information neighborhood and votes for $y$ otherwise. Unfortunately, this is also not a viable option as shown in Example 3.8 below.

The process of finding the optimal communication strategy under the empty network is additionally simplified by the fact that restricting attention to anonymous communication strategies is without loss of generality (Kerman et al., 2020). For a general network structure it follows trivially that assuming anonymity is no longer without loss.

\subsection{Truth-Telling in the Sender's Preferred State}

In the standard single-receiver case, as well as most multiple-receiver models, telling the truth to the receiver(s) with probability 1 in the sender's preferred state $(X)$ is optimal. This common result ceases to hold in general in the current setup, and this is illustrated by Example 3.8. To help with the analysis we introduce a lemma, which establishes that the maximum number of messages a communication strategy requires to achieve the optimal value is at most equal to its number of anchors plus one.

Lemma 3.7. Let $g \in G(N)$ and $\pi \in \Pi$. There exists $\pi^{\prime} \in \Pi$ with $\left|A n\left(\pi^{\prime}\right)\right|=|A n(\pi)|$ such that for each $i \in N$ it holds that $\left|S_{i}^{\pi^{\prime}}\right| \leq\left|A n\left(\pi^{\prime}\right)\right|+1$ and $V_{k}^{\pi^{\prime}}(g)=V_{k}^{\pi}(g)$.

Example 3.8. Let $|N|=6, \lambda^{0}(X)=1 / 3$, and $k=4$. Consider the following network $g$ and the communication strategy $\pi$ which has one anchor and sends $x$ to all receivers with probability 1 in state $X$ :

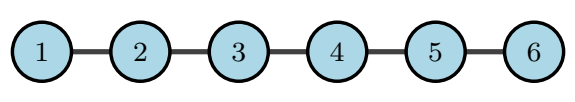

\begin{tabular}{c|cc}
$\pi$ & $\omega=X$ & $\omega=Y$ \\
\hline$(x, x, x, x, x, x)$ & 1 & 0 \\
$(y, x, x, x, x, x)$ & 0 & $\frac{1}{4}$ \\
$(x, x, x, x, x, y)$ & 0 & $\frac{1}{4}$ \\
$(y, y, y, y, y, y)$ & 0 & $\frac{1}{2}$
\end{tabular}

\footnotetext{
${ }^{3}$ Proposition 5.2 in the next section shows that in fact the structure of the communcation strategy $\pi^{\prime}$ is optimal and results in the optimal value which can be achieved in any star network.
} 
The value of $\pi$ is given by $V_{4}^{\pi}(g)=2 / 3$. This is equal to $V^{p}$, the value of the optimal public communication strategy.

Now consider communication strategy $\pi^{\prime}$, which has two anchor signals and support:

\begin{tabular}{c|cc}
$\pi^{\prime}$ & $\omega=X$ & $\omega=Y$ \\
\hline$(x, x, x, x, x, x)$ & $\frac{1}{2}$ & 0 \\
$(y, y, y, y, y, y)$ & $\frac{1}{2}$ & 0 \\
$(x, x, x, y, y, y)$ & 0 & $\frac{1}{4}$ \\
$(y, x, x, x, x, x)$ & 0 & $\frac{1}{4}$ \\
$(y, y, y, y, y, x)$ & 0 & $\frac{1}{4}$ \\
$(y, x, y, x, y, x)$ & 0 & $\frac{1}{4}$
\end{tabular}

$$
\begin{aligned}
\operatorname{supp}\left(\sigma^{g}\right)=\{ & \left(\frac{1}{2}, \frac{1}{2}, \frac{1}{2}, \frac{1}{2}, \frac{1}{2}, \frac{1}{2}\right),\left(\frac{1}{2}, \frac{1}{2}, \frac{1}{2}, \frac{1}{2}, \frac{1}{2}, \frac{1}{2}\right), \\
& \left(\frac{1}{2}, \frac{1}{2}, 0,0, \frac{1}{2}, \frac{1}{2}\right),\left(0,0, \frac{1}{2}, \frac{1}{2}, \frac{1}{2}, \frac{1}{2}\right), \\
& \left.\left(\frac{1}{2}, \frac{1}{2}, \frac{1}{2}, \frac{1}{2}, 0,0\right),(0,0,0,0,0,0)\right\} .
\end{aligned}
$$

The value is $V_{4}^{\pi^{\prime}}(g)=1 / 3 \cdot 1+2 / 3 \cdot 3 / 4=5 / 6>2 / 3$, which shows that the sender can improve upon $\pi$ by garbling information in state $X{ }^{4}$

It is important that $V_{4}^{\pi^{\prime}}(g)$ cannot be achieved in a strategy with only one anchor. To prove this, assume the contrary. By Lemma 3.7, there exists $\pi^{\prime \prime} \in \Pi$ such that for every $i \in N$ it holds that $\left|S_{i}^{\pi^{\prime \prime}}\right|=2$ and $V_{4}^{\pi^{\prime \prime}}(g)=V_{4}^{\pi^{\prime}}(g)=5 / 6$. Assume without loss of generality that for each $i \in N, S_{i}^{\pi^{\prime \prime}}=\{x, y\}$. As truth-telling in state $X$ requires, let $\bar{x}=(x, \ldots, x)$ be the anchor and let $\pi^{\prime \prime}(\bar{x} \mid X)=1$. Let $t \in S^{\pi^{\prime \prime}}$ with $t \neq \bar{x}$. If for any $i \in N$ it holds that $t_{i} \neq x$, then for any $j \in \bar{N}_{i}(g)$ we have $\alpha_{j}^{\pi^{\prime \prime}, g}\left(t_{j}(g)\right)=y$ and thus, if $i \in\{2,3,4,5\}$, then $\alpha^{\pi^{\prime \prime}, g}(t)=y$, i.e. $t$ does not implement $x$. Thus, in signals that implement $x$, only receivers 1 and 6 can observe a message different than $x$. In this case, an optimal communication strategy is $\pi$ given above, which has value $2 / 3$, contradicting that $V_{4}^{\pi^{\prime \prime}}(g)=5 / 6$. Therefore, truth-telling in state $X$ is not optimal in general.

Recall the naive straightforwardness extension in the previous section; it is not violated by $\pi$. Moreover, $\pi$ is optimal among communication strategies which satisfy this definition. Since we can improve upon $\pi$ with the "non-naively straightforward" $\pi^{\prime}$, it follows that the naive extension is not optimal either. The feature which makes $\pi^{\prime}$ better than $\pi$ is that apart from the different personal messages within signals, it uses the differences in the messages between the two anchors to create more variation in the strategy.

\section{General Observations}

As shown, many of the usual simplifying assumptions in the empty network Bayesian persuasion model do not generally extend to non-empty networks. Nevertheless, there are optimality results which can be recovered and they are the subject of this section.

\footnotetext{
${ }^{4}$ Note that $\pi^{\prime}$ is optimal on $g$ (as implied by Proposition 4.1 in the next section). The same value could have been achieved with at most three messages (cf. Lemma 3.7) by sending a different message in the signal which does not implement $x$. However, it is possible to create this signal such that it is distinguished from both anchors by all nodes with only two messages.
} 


\subsection{The Network Does not Benefit the Sender}

We start by showing that having a network structure cannot be strictly beneficial for the sender. More precisely, for any communication strategy the optimal value under the empty network $g_{0}$ is at least as good as the optimal value under any other network $g$ with the same number of nodes. This insight was already hinted at in Example 3.2. The simple logic behind this observation is that in a non-empty network the vote of every node is determined not by the individual messages of every receiver but by his whole neighborhood. However, the information a receiver gathers from a communication strategy in a non-empty network can be replicated in an empty network of the same size.

Proposition 4.1. Let $g \in G(N)$. For any $\pi \in \Pi$ it holds that $V_{k}^{\pi}(g) \leq V_{k}^{n}$.

Intuitively, the reason the empty network value weakly dominates the value of any other network with the same number of nodes is that the sender can target at least as many winning coalitions under the empty network as under any non-empty network. However, this might not hold when comparing two non-empty networks, as shown in Section 6.

\subsection{Networks with Isolated Nodes}

This section shows that with sufficiently many isolated nodes the network has no effect on what the sender can achieve. In particular, if $k \geq n / 2$ and if the number of singletons in a network is at least $k$, then the sender can achieve the empty network optimal value, irrespective of the connections between the remaining nodes.

Example 4.2. Let $|N|=9, \lambda^{0}(X)=1 / 3$, and $k=5$. Consider network $g$ below, the communication strategy $\pi$ and the support of its induced distribution. Note that $2 k=$ $10>9=n$, so the singleton nodes are equal to $k$ and are a simple majority.

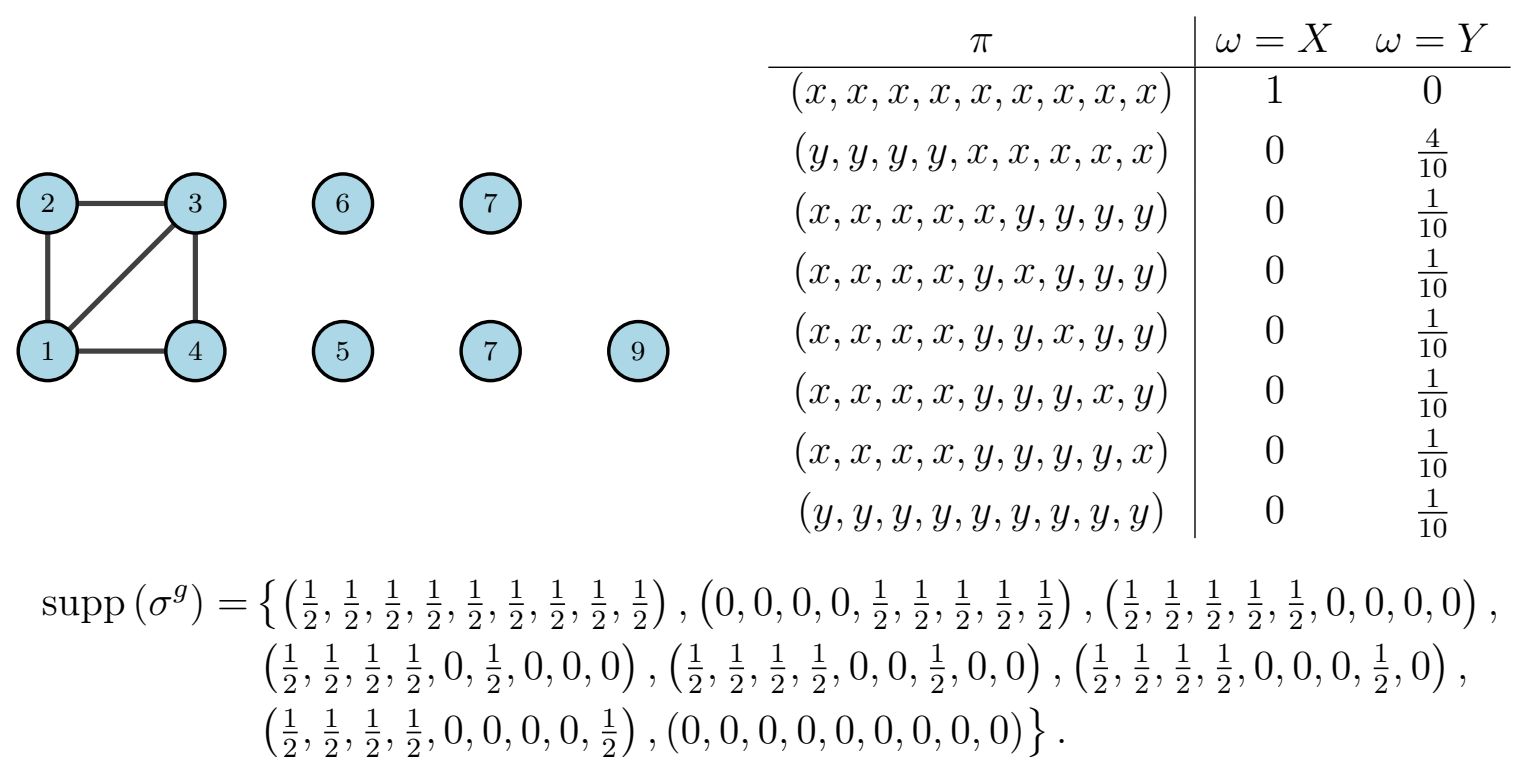


The value is $V_{5}^{\pi}(g)=1 / 3 \cdot 1+2 / 3 \cdot 9 / 10=14 / 15=V_{5}^{9}$, the value for persuading $k=5$ out of $n=9$ in an empty network. Observe that this value can be achieved irrespective of the connections between nodes 1-4, as these nodes are always treated uniformly.

Proposition 4.3. Let $g \in G(N)$ and $\left|\left\{i \in N: \delta_{i}^{g}=0\right\}\right| \geq k$. If $k \geq n / 2$, then there exists $\pi \in \Pi$ such that $V_{k}^{\pi}(g)=V_{k}^{n}$.

\subsection{Networks Consisting of Complete Components}

If two nodes $i$ and $j$ in a network $g$ have exactly the same neighborhood, $\bar{N}_{i}(g)=\bar{N}_{j}(g)$, then the sets of signals they consider possible under any communication strategy $\pi$ are the same, i.e. $A_{i}^{\pi}(g, s)=A_{j}^{\pi}(g, s)$. This implies that they always have the same posterior belief and vote for the same alternative, i.e. for every $s \in S^{\pi}$ it holds that $\alpha_{i}^{\pi, g}\left(s_{i}(g)\right)=$ $\alpha_{j}^{\pi, g}\left(s_{j}(g)\right)$. Therefore, in such cases we can restrict attention to communication strategies which send the same message to $i$ and $j$. In particular, the information that the sender wants to provide to the neighbours of $i$ and $j$ by sending different messages to $i$ and $j$ can also be provided to the neighbours by sending the same message to $i$ and $j$ within the same signal. Note that in this case $i$ and $j$ can still observe different messages between signals. This logic is formalized in Proposition 4.4, which is particularly relevant for members of a complete component because they always share the same information neighorhood.

Proposition 4.4. Let $\pi \in \Pi$ and let $g \in G(N)$ and $i, j \in N$ be such that $\bar{N}_{i}(g)=\bar{N}_{j}(g)$. Then there exists $\pi^{\prime} \in \Pi$ such that for any $s \in S^{\pi^{\prime}}, s_{i}=s_{j}$ and $V_{k}^{\pi^{\prime}}(g)=V_{k}^{\pi}(g)$.

As every receiver in a complete network has the same information neighborhood, an immediate corollary to Proposition 4.4 follows.

Corollary 4.5. Let $g \in G(N)$ be a complete network. Then there exists an optimal $\pi \in \Pi$ such that $V_{k}^{\pi}(g)=V^{p}$.

So, the optimal communication strategy in a complete network is public as it is optimal for the sender to treat all receivers within a signal uniformly by sending them same message.

Proposition 4.4 naturally extends to networks of disjoint complete components. Treating all nodes within a component uniformly in every signal makes this setup similar to an empty network with fewer nodes where every node has a different number of votes.

Example 4.6. Let $|N|=9, \lambda^{0}(X)=1 / 3$, and $k=5$. Consider the following network $g$ with three complete components and the communication strategy $\pi$ :
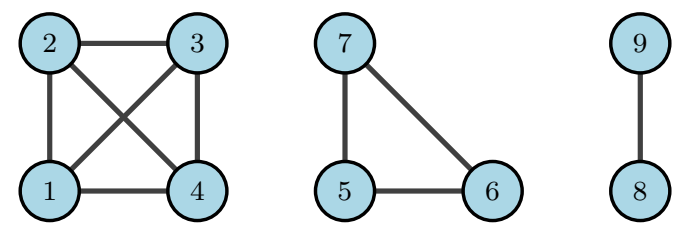

\begin{tabular}{c|cc}
$\pi$ & $\omega=X$ & $\omega=Y$ \\
\hline$(x, x, x, x, x, x, x, x, x)$ & 1 & 0 \\
$(y, y, y, y, x, x, x, x, x)$ & 0 & $\frac{1}{4}$ \\
$(x, x, x, x, y, y, y, x, x)$ & 0 & $\frac{1}{4}$ \\
$(x, x, x, x, x, x, x, y, y)$ & 0 & $\frac{1}{4}$ \\
$(y, y, y, y, y, y, y, y, y)$ & 0 & $\frac{1}{4}$
\end{tabular}


Here, selecting any component to receive a $y$ message leaves enough nodes $(5,6$, or 7$)$ to implement $x$. This results in:

$$
\begin{aligned}
\operatorname{supp}\left(\sigma^{g}\right)= & \left\{\left(\frac{1}{2}, \frac{1}{2}, \frac{1}{2}, \frac{1}{2}, \frac{1}{2}, \frac{1}{2}, \frac{1}{2}, \frac{1}{2}, \frac{1}{2}\right),\left(0,0,0,0, \frac{1}{2}, \frac{1}{2}, \frac{1}{2}, \frac{1}{2}, \frac{1}{2}\right),\left(\frac{1}{2}, \frac{1}{2}, \frac{1}{2}, \frac{1}{2}, 0,0,0, \frac{1}{2}, \frac{1}{2}\right),\right. \\
& \left.\left(\frac{1}{2}, \frac{1}{2}, \frac{1}{2}, \frac{1}{2}, \frac{1}{2}, \frac{1}{2}, \frac{1}{2}, 0,0\right),(0,0,0,0,0,0,0,0,0)\right\} .
\end{aligned}
$$

The value is $V_{5}^{\pi}(g)=1 / 3 \cdot 1+2 / 3 \cdot 3 / 4=5 / 6=V_{2}^{3}$, the value for persuading $k=2$ out of $n=3$ nodes in the empty network and it is clear that no higher value can be achieved.

However, this is not necessarily the case if selecting a different number of components in different signals can still fulfil the quota, as this allows for much more variation. For example, when nodes 5,6,7 are singletons strategy $\pi^{\prime}$ can be applied.
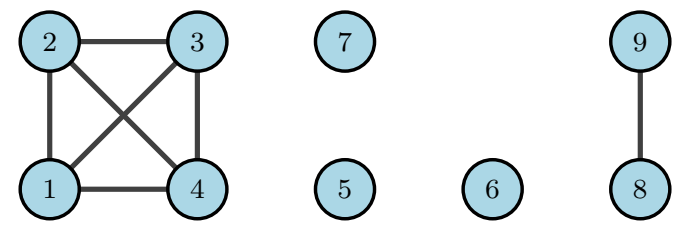

\begin{tabular}{c|cc}
$\pi^{\prime}$ & $\omega=X$ & $\omega=Y$ \\
\hline$(x, x, x, x, x, x, x, x, x)$ & 1 & 0 \\
$(y, y, y, y, x, x, x, x, x)$ & 0 & $\frac{1}{3}$ \\
$(x, x, x, x, y, x, y, y, y)$ & 0 & $\frac{1}{6}$ \\
$(x, x, x, x, x, y, y, y, y)$ & 0 & $\frac{1}{6}$ \\
$(x, x, x, x, y, y, x, y, y)$ & 0 & $\frac{1}{6}$ \\
$(y, y, y, y, y, y, y, y, y)$ & 0 & $\frac{1}{6}$
\end{tabular}

In this case the support of $\pi^{\prime}$ is:

$$
\begin{aligned}
\operatorname{supp}\left(\sigma^{g}\right)= & \left\{\left(\frac{1}{2}, \frac{1}{2}, \frac{1}{2}, \frac{1}{2}, \frac{1}{2}, \frac{1}{2}, \frac{1}{2}, \frac{3}{5}, \frac{3}{5}\right),\left(0,0,0,0, \frac{1}{2}, \frac{1}{2}, \frac{1}{2}, \frac{3}{5}, \frac{3}{5}\right),\left(\frac{1}{2}, \frac{1}{2}, \frac{1}{2}, \frac{1}{2}, 0, \frac{1}{2}, 0,0,0\right),\right. \\
& \left.\left(\frac{1}{2}, \frac{1}{2}, \frac{1}{2}, \frac{1}{2}, \frac{1}{2}, 0,0,0,0\right),\left(\frac{1}{2}, \frac{1}{2}, \frac{1}{2}, \frac{1}{2}, 0,0, \frac{1}{2}, 0,0\right),(0,0,0,0,0,0,0,0,0)\right\}
\end{aligned}
$$

The value is $V_{5}^{\pi^{\prime}}(g)=1 / 3 \cdot 1+2 / 3 \cdot 5 / 6=8 / 9>5 / 6$.

To formalize the logic of the example, given a network $g$, let $C(g)$ be the set of all components of $g$. For $|C(g)|=\ell$, let $\mathfrak{C}^{q}$ be the set of all subsets of components, where each subset has cardinality $q$. That is, $\mathfrak{C}^{q}=\left\{\mathcal{C}^{\prime} \subseteq C(g):\left|\mathcal{C}^{\prime}\right|=q\right\}$.

A network $g \in G(N)$ is connected if for any $i, j \in N$ there is a path between $i$ and $j$.

Proposition 4.7. Let $g \in G(N)$ be a disconnected network consisting of $\ell$ complete components of respective sizes $c_{1}, c_{2}, \ldots, c_{\ell} \in C(g)$. If $q \in \mathbb{N}$ is such that for each $\mathcal{C}^{\prime} \in \mathfrak{C}^{q}$ it holds that $\sum_{C \in \mathcal{C}^{\prime}}|C| \geq k$, then there exists an optimal $\pi \in \Pi$ such that $V_{k}^{\pi}(g)=V_{q}^{\ell}$.

So, if a network consists of $\ell$ complete disjoint components and combining the same number of components $q$ fulfils the quota, it is possible to construct a strategy with value equal to the optimal value of persuading $q$ out of $\ell$ agents. This follows directly from the uniform treatment of all nodes within the same component as per Proposition 4.4. 


\section{Optimality in Specific Network Structures}

This section provides optimal strategies for networks which frequently appear in the literature. Every subsection deals with different network types and starts with an example conveying the intuition which drives the more general result and the main mechanisms to construct the optimal strategies. This is followed by a formal statement of the result.

\subsection{Star Networks}

A network $g \in G(N)$ is a star if there exists $j \in N$ such that for any $i \in N$ with $i \neq j$ it holds that $g_{i j}=1$ and for any $\ell \in N$ with $\ell \neq j$ it holds that $g_{i \ell}=0$. The star presents a situation in which the optimal value under the empty network cannot be achieved.

Before proceeding to the formal statement about star networks, consider the example below, which bears similarity to Example 3.6. The communication strategy employed keeps the center's message the same across all signals which implement $x$ while varying the periphery nodes' messages in the same way as in the empty network and thus achieves value equal to $V_{k}^{n-1}$. This is less than $V_{k}^{n}$ and so the optimal value in the empty network with private communication is not achieved in this case.

Example 5.1. Let $|N|=5, \lambda^{0}(X)=1 / 3$, and $k=3$. Consider the following network $g$, the communication strategy $\pi$ and its support:

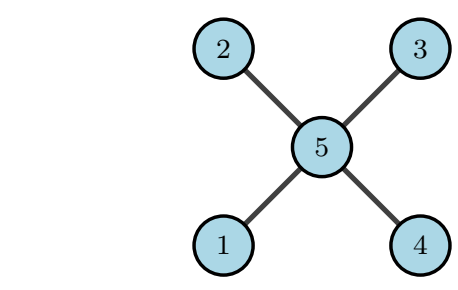

\begin{tabular}{c|cc}
$\pi$ & $\omega=X$ & $\omega=Y$ \\
\hline$(x, x, x, x, x)$ & 1 & 0 \\
$(y, x, x, x, x)$ & 0 & $\frac{1}{6}$ \\
$(x, y, x, x, x)$ & 0 & $\frac{1}{6}$ \\
$(x, x, y, x, x)$ & 0 & $\frac{1}{6}$ \\
$(x, x, x, y, x)$ & 0 & $\frac{1}{6}$ \\
$(y, y, y, y, y)$ & 0 & $\frac{1}{3}$
\end{tabular}

$$
\begin{aligned}
\operatorname{supp}\left(\sigma^{g}\right)= & \left(\frac{1}{2}, \frac{1}{2}, \frac{1}{2}, \frac{1}{2}, 1\right),\left(0, \frac{1}{2}, \frac{1}{2}, \frac{1}{2}, 0\right),\left(\frac{1}{2}, 0, \frac{1}{2}, \frac{1}{2}, 0\right),\left(\frac{1}{2}, \frac{1}{2}, 0, \frac{1}{2}, 0\right),\left(\frac{1}{2}, \frac{1}{2}, \frac{1}{2}, 0,0\right), \\
& (0,0,0,0,0)\} .
\end{aligned}
$$

The value is $V_{3}^{\pi}(g)=1 / 3 \cdot 1+2 / 3 \cdot 2 / 3=7 / 9=V_{3}^{4}$.

Proposition 5.2. Let $g \in G(N)$ be a star and let $k<n$. Then there exists an optimal $\pi \in \Pi$ such that $V_{k}^{\pi}(g)=V_{k}^{n-1}$.

In the optimal communication strategy specified in the proof of Proposition 5.2 the center node observes the same message in all signals which implement $x$. It is possible to extrapolate this logic to all networks which have nodes that are connected to all other nodes. In the limit case (the complete network), this corresponds to sending the same messages to all receivers within a signal for every signal, i.e. a public communication strategy. ${ }^{5}$

\footnotetext{
${ }^{5}$ Similarly, when $n=k$ in a star, optimal communication is public.
} 


\subsection{Regular Networks}

A network $g$ is regular if for any $i, j \in N$ it holds that $\delta_{i}^{g}=\delta_{j}^{g}=\delta$. Regular networks are a natural extension of the situation described by Theorem 3.1 and Corollary 4.5, as the empty network is also regular. A regular connected network with $\delta=2$ is a circle.

Example 5.3. Let $|N|=9, \lambda^{0}(X)=1 / 3$, and $k=6$. Consider the following network $g$, the communication strategy $\pi$ and its support:

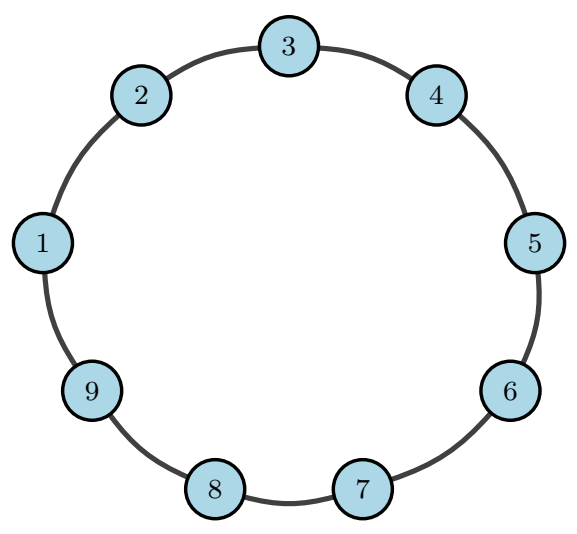

\begin{tabular}{c|cc}
$\pi$ & $\omega=X$ & $\omega=Y$ \\
\hline$(x, x, x, x, x, x, x, x, x)$ & 1 & 0 \\
$(y, x, x, x, x, x, x, x, x)$ & 0 & $\frac{1}{12}$ \\
$(x, y, x, x, x, x, x, x, x)$ & 0 & $\frac{1}{12}$ \\
$(x, x, y, x, x, x, x, x, x)$ & 0 & $\frac{1}{12}$ \\
$(x, x, x, y, x, x, x, x, x)$ & 0 & $\frac{1}{12}$ \\
$(x, x, x, x, y, x, x, x, x)$ & 0 & $\frac{1}{12}$ \\
$(x, x, x, x, x, y, x, x, x)$ & 0 & $\frac{1}{12}$ \\
$(x, x, x, x, x, x, y, x, x)$ & 0 & $\frac{1}{12}$ \\
$(x, x, x, x, x, x, x, y, x)$ & 0 & $\frac{1}{12}$ \\
$(x, x, x, x, x, x, x, x, y)$ & 0 & $\frac{1}{12}$ \\
$(y, y, y, y, y, y, y, y, y)$ & 0 & $\frac{1}{4}$
\end{tabular}

$$
\begin{aligned}
\operatorname{supp}\left(\sigma^{g}\right)= & \left(\frac{1}{2}, \frac{1}{2}, \frac{1}{2}, \frac{1}{2}, \frac{1}{2}, \frac{1}{2}, \frac{1}{2}, \frac{1}{2}, \frac{1}{2}\right),\left(0,0, \frac{1}{2}, \frac{1}{2}, \frac{1}{2}, \frac{1}{2}, \frac{1}{2}, \frac{1}{2}, 0\right), \\
& \left(0,0,0, \frac{1}{2}, \frac{1}{2}, \frac{1}{2}, \frac{1}{2}, \frac{1}{2}, \frac{1}{2}\right),\left(\frac{1}{2}, 0,0,0, \frac{1}{2}, \frac{1}{2}, \frac{1}{2}, \frac{1}{2}, \frac{1}{2}\right),\left(\frac{1}{2}, \frac{1}{2}, 0,0,0, \frac{1}{2}, \frac{1}{2}, \frac{1}{2}, \frac{1}{2}\right), \\
& \left(\frac{1}{2}, \frac{1}{2}, \frac{1}{2}, 0,0,0, \frac{1}{2}, \frac{1}{2}, \frac{1}{2}\right),\left(\frac{1}{2}, \frac{1}{2}, \frac{1}{2}, \frac{1}{2}, 0,0,0, \frac{1}{2}, \frac{1}{2}\right),\left(\frac{1}{2}, \frac{1}{2}, \frac{1}{2}, \frac{1}{2}, \frac{1}{2}, 0,0,0, \frac{1}{2}\right), \\
& \left.\left(\frac{1}{2}, \frac{1}{2}, \frac{1}{2}, \frac{1}{2}, \frac{1}{2}, \frac{1}{2}, 0,0,0\right),\left(0, \frac{1}{2}, \frac{1}{2}, \frac{1}{2}, \frac{1}{2}, \frac{1}{2}, \frac{1}{2}, 0,0\right),(0,0,0,0,0,0,0,0,0)\right\} .
\end{aligned}
$$

Each receiver observes $x$ with probability 1 in state $X$. Hence, sending $y$ to a receiver in state $Y$ implies that 3 receivers vote for $y$. So, in a signal implementing $x$ in state $Y$ exactly 6 receivers are persuaded and $V_{6}^{\pi}(g)=1 / 3 \cdot 1+2 / 3 \cdot 9 / 12=5 / 6=V_{6}^{9}$.

Proposition 5.4. Let $g \in G(N)$ be a circle and let $k<n-2$. Then there exists $\pi \in \Pi$ such that $V_{k}^{\pi}(g)=V_{k}^{n}$.

Proposition 5.4 formalizes the observation in Example 5.3 and establishes that if the quota is less than $n-2$, the optimal value under the empty network can be achieved on a circle.

A network which combines many properties of circle and star networks is the wheel: formally, a network $g \in G(N)$ is a wheel if there exists $j \in N$ such that for any $i \in N$ with $i \neq j$ it holds that: (i) $g_{i j}=1$, (ii) $\delta_{i}^{g}=3$, and (iii) for any $\ell \in N, \ell \neq j$, and $\ell \neq i$ there is a path between $i$ and $\ell$ which does not include $j$.

The reasoning behind sending the same message to the center node in all signals which implement $x$ used for the star also applies to the wheel. In this case, the sender can treat 
the network as if it is a circle to achieve the optimal value provided that the quota is less than $n-3$. This observation is stated as a corollary to Propositions 5.2 and 5.4.

Corollary 5.5. Let $g \in G(N)$ be a wheel and let $k<n-3$. Then there exists an optimal $\pi \in \Pi$ such that $V_{k}^{\pi}(g)=V_{k}^{n-1}$.

Proposition 5.6. Let $g \in G(N)$ be a regular network with degree $\delta$ and let $k=n-1-\delta$. Then there exists $\pi \in \Pi$ such that $V_{k}^{\pi}(g)=V_{k}^{n}$.

Proposition 5.6 shows that if $k=n-1-\delta$ the logic used for the circle can be applied to every regular network to obtain the optimal value. The following example illustrates that extending the reasoning in the proposition to $k<n-1-\delta$ is a non-trivial task.

Example 5.7. Consider the following regular networks $g_{1}, g_{2}$ and $g_{3}$, all with degree 3 .

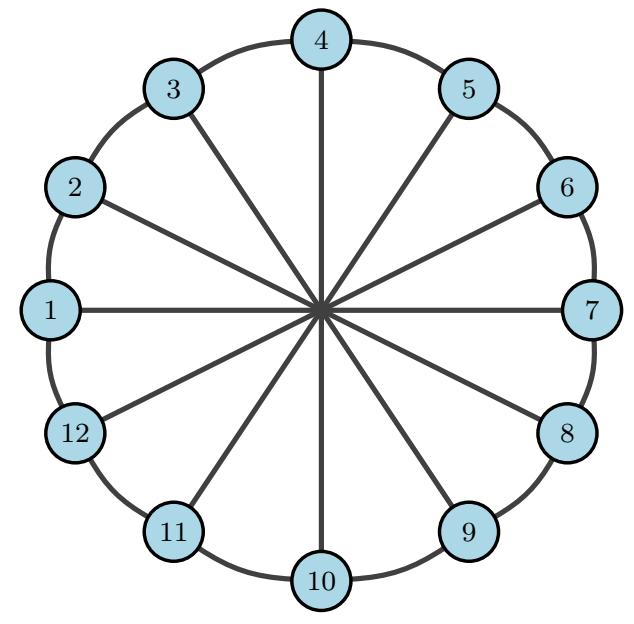

Network $g_{1}$

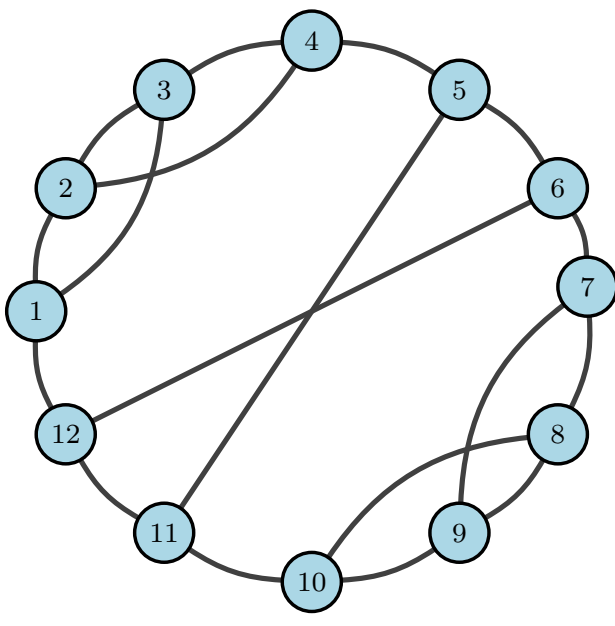

Network $g_{2}$

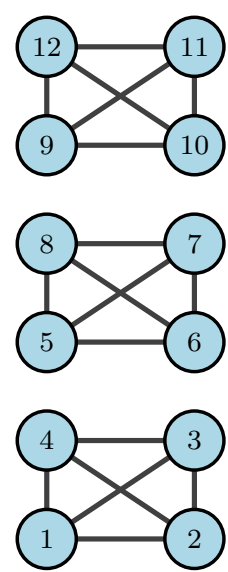

Network $g_{3}$

Let $\lambda^{0}(X)=1 / 3$. By Proposition 5.6, the optimal value under the empty network can be achieved in all three networks for $k=8$. However, if $k=6$ we can easily construct a communication strategy with value equal to $V_{6}^{12}=V_{1}^{2}=1$ for $g_{1}$ and $g_{2}$ (similar to the construction in Example 5.3), but not for $g_{3}$. Because the network is disconnected and each component is complete, persuading exactly 6 receivers is impossible due to Proposition 4.4 as receivers in a complete component all vote for the same alternative. In particular, any signal that implements $x$ has at least 8 receivers who vote for $x$, implying that the value 1 cannot be achieved.

Decreasing the quota to $k=4$, it is once again easy to construct optimal strategies for $g_{1}$ and $g_{3}$, but an optimal strategy for $g_{2}$ will clearly have a different structure.

It is important to note that the conditions in Proposition 5.4 and 5.6 are sufficient, but not necessary. The next example provides a network which satisfies neither of them but the optimal communication strategy on it still achieves the maximum value. 
Example 5.8. Let $|N|=4, \lambda^{0}(X)=1 / 3$. Consider the following network $g$ and the communication strategies $\pi$ for $k=2$ and $\pi^{\prime}$ for $k=3$ with corresponding supports:

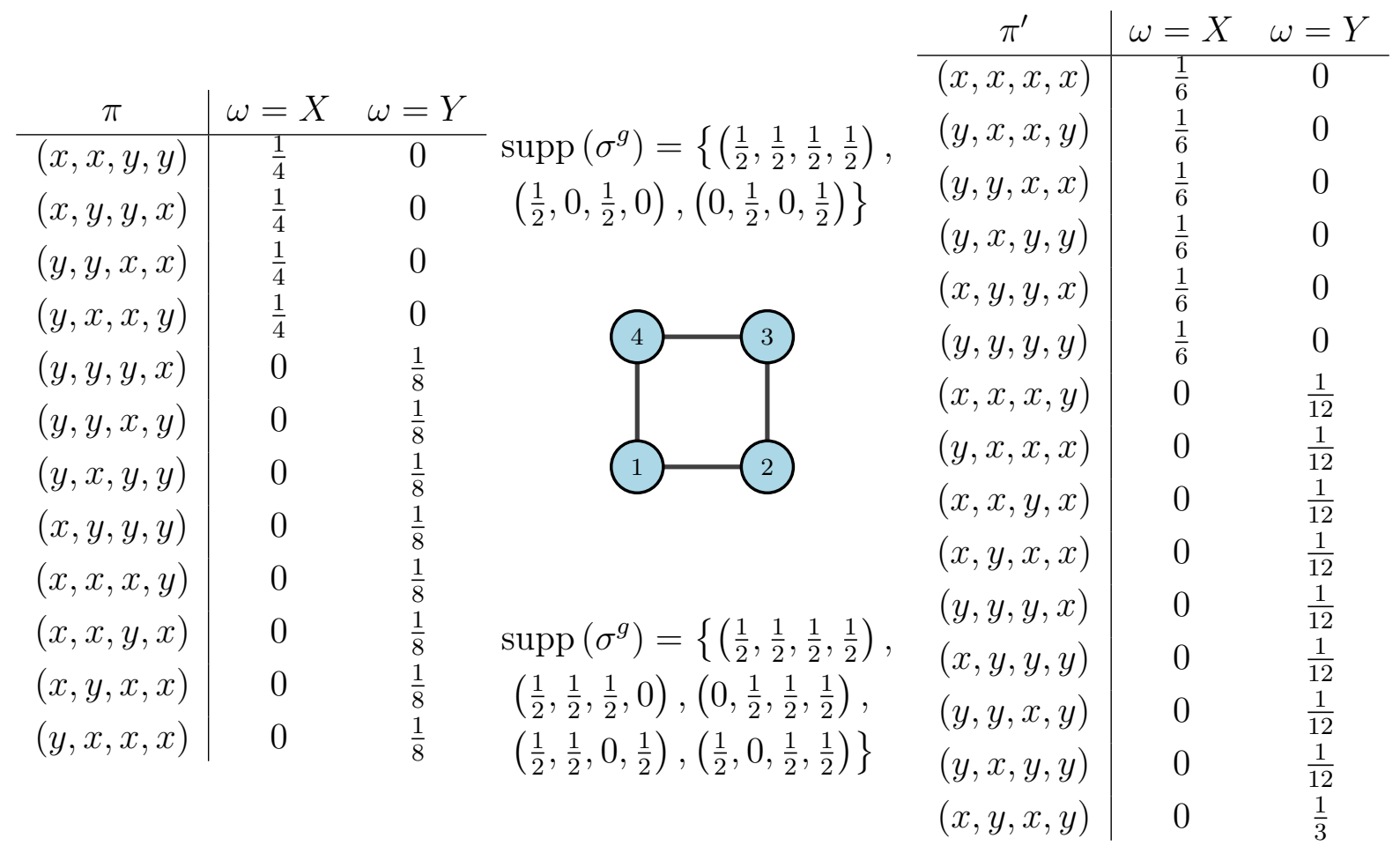

where in the first support the first four signals in $\pi$ produce the first posterior, the oddnumbered remaining signals have the second one and the even-numbered remaining signals have the third posterior. As all signals in $\pi$ implement $x$, the value is $V_{2}^{\pi}(g)=1=V_{2}^{4}$, while for $\pi^{\prime}$ it is $V_{3}^{\pi^{\prime}}(g)=7 / 9=V_{3}^{4}$. Following the same logic as in Example 3.8 it is possible to see that in both cases the values cannot be achieved by simpler strategies, i.e. ones with fewer total signals or fewer anchors. The underlying structures also cannot be readily extrapolated to optimal strategies for the general case of $k=n-2$ or $k=n-1$ on a circle, as such an extrapolation would require using more than two messages.

\subsection{Irregular Networks}

Moving away from regular networks, it is possible to derive intuitive results for irregular networks which have some sufficient degree of symmetry like in the example below.

Example 5.9. Let $|N|=36, \lambda^{0}(X)=1 / 3$, and $k=27$. Consider network $g$ below. 


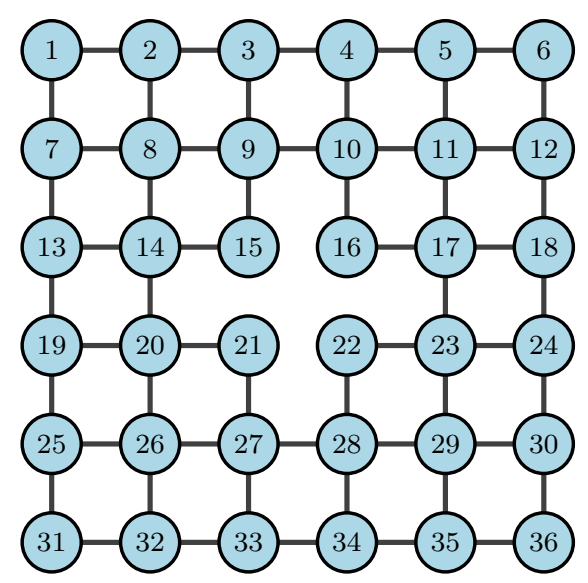

\begin{tabular}{c|cc}
$\pi$ & $\omega=X$ & $\omega=Y$ \\
\hline $\bar{x}$ & 1 & 0 \\
$s^{1}$ & 0 & $\frac{1}{6}$ \\
$s^{2}$ & 0 & $\frac{1}{6}$ \\
$s^{3}$ & 0 & $\frac{1}{6}$ \\
$s^{4}$ & 0 & $\frac{1}{6}$ \\
$\bar{y}$ & 0 & $\frac{1}{3}$
\end{tabular}

Notice that there are no links between nodes 15 and 16, 15 and 21, 16 and 22, and 21 and 22. Further, it is important that while the network shows some symmetry, it is not regular as there are nodes with degrees 2,3 , and 4 . Additionally, exactly $1 / 4$ of it can be covered by sending a message $y$ to specific agents in one of the quadrants. In particular, sending $y$ to receivers $2,7,8$, and 15 makes receivers in the NW quadrant (and no one else) observe at least one $y$ in their information neighborhood. Define signals:

(i) $s^{1} \in S$ such that for each $i \in N, s_{i}^{1}=y$ if $i \in\{2,7,8,15\}$ and $s_{i}^{1}=x$ otherwise,

(ii) $s^{2} \in S$ such that for each $i \in N, s_{i}^{2}=y$ if $i \in\{5,11,12,16\}$ and $s_{i}^{1}=x$ otherwise,

(iii) $s^{3} \in S$ such that for each $i \in N, s_{i}^{3}=y$ if $i \in\{21,25,26,32\}$ and $s_{i}^{1}=x$ otherwise,

(iv) $s^{4} \in S$ such that for each $i \in N, s_{i}^{4}=y$ if $i \in\{22,29,30,35\}$ and $s_{i}^{1}=x$ otherwise.

Consider communication strategy $\pi$ and note that $s^{1}, s^{2}, s^{3}$, and $s^{4}$ make receivers in the NW, NE, SW, and SE quadrant respectively vote for $y . V_{27}^{\pi}(g)=V_{27}^{36}=V_{3}^{4}=7 / 9$.

Proposition 5.10. Let $g \in G(N)$ be a network and let $q \in \mathbb{N}$ be a common factor of $n$ and $k$. If there exist $n / q$ disjoint sets of nodes $O_{1}, \ldots, O_{n / q} \subseteq N$ such that for each $j \in\{1, \ldots, n / q\}$ it holds that $\left|O_{j}\right|=q$ and for each $O_{j} \subseteq N$ there exists $L_{j} \subseteq O_{j}$ with $\cup_{i \in L_{j}} \bar{N}_{i}(g)=O_{j}$, then there exists $\pi \in \Pi$ with $V_{k}^{\pi}(g)=V_{k}^{n}$.

It is important to notice that the proposition presents only a sufficient condition. It generalizes the observation from Example 5.9 that if equal-sized parts of the network can observe at least one $y$ message in their information neighborhood in different signals this can be leveraged to get the optimal value, irrespective of regularity. Finally, it also extends the underlying logic behind Proposition 5.4 and 5.6 to irregular networks. 


\subsubsection{Line Networks}

The line network is a notable case of a network which in some cases can be divided in the way described in Proposition 5.10.

Example 5.11. Let $|N|=9, \lambda^{0}(X)=1 / 3$, and $k=6$. Consider the following network $g$, the communication strategy $\pi \in \Pi$ and its support:

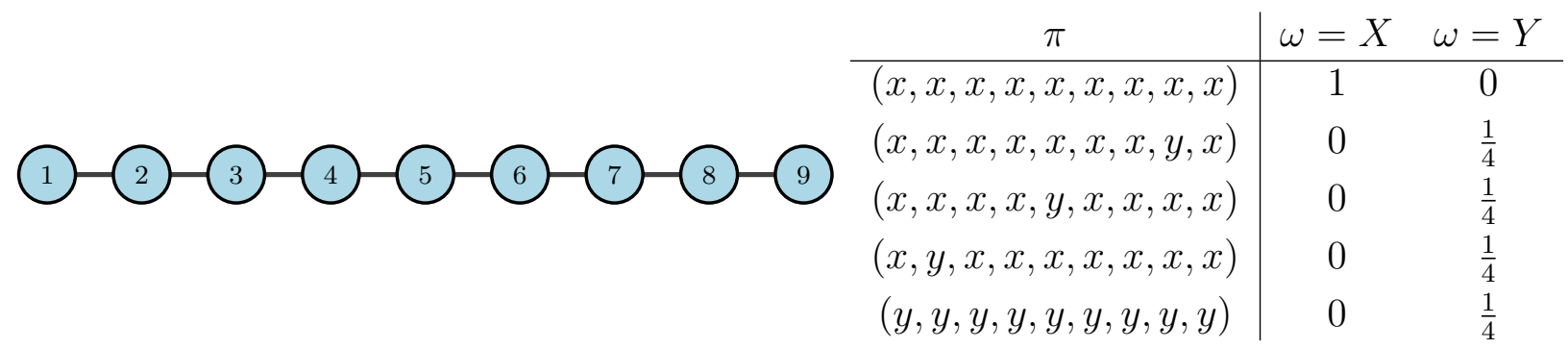

$$
\begin{aligned}
& \operatorname{supp}\left(\sigma^{g}\right)=\left\{\left(\frac{1}{2}, \frac{1}{2}, \frac{1}{2}, \frac{1}{2}, \frac{1}{2}, \frac{1}{2}, \frac{1}{2}, \frac{1}{2}, \frac{1}{2}\right),\left(\frac{1}{2}, \frac{1}{2}, \frac{1}{2}, \frac{1}{2}, \frac{1}{2}, \frac{1}{2}, 0,0,0\right),\left(\frac{1}{2}, \frac{1}{2}, \frac{1}{2}, 0,0,0, \frac{1}{2}, \frac{1}{2}, \frac{1}{2},\right)\right. \text {, } \\
& \left.\left(0,0,0, \frac{1}{2}, \frac{1}{2}, \frac{1}{2}, \frac{1}{2}, \frac{1}{2}, \frac{1}{2}\right),(0,0,0,0,0,0,0,0,0)\right\} \text {. }
\end{aligned}
$$

The value is $V_{6}^{\pi}(g)=1 / 3 \cdot 1+2 / 3 \cdot 3 / 4=5 / 6=V_{6}^{9}$. Interestingly, by Proposition 5.10, $\pi$ is a simpler alternative to the communication strategy in Example 5.3. It preserves the value $V_{6}^{9}$ also in the case of a circle (which has an added link with respect to $g$ ).

The following corollary to Proposition 5.10 states that line networks with a common factor 2 or 3 for $n$ and $k$ can achieve the optimal value by building optimal strategies following the pattern of Example 3.8 or 5.11 and hence it is presented without proof.

Corollary 5.12. If $g \in G(N)$ is a line and if for $\alpha, \beta \in \mathbb{N}$ : (i) $k=3 \alpha, n=3 \beta$ or (ii) $k=2 \alpha, n=2 \beta$, then there exists $\pi \in \Pi$ such that $V_{k}^{\pi}(g)=V_{k}^{n}$.

\section{Adding Links}

Section 3 showed that adding a link to the network while keeping the communication strategy fixed can leave the sender better or worse off. Example 3.3 demonstrates how for a suboptimal communication strategy adding a link benefits the sender. Additionally, Example 5.11 illustrates that making a circle from a line can preserve the optimal value.

When the communication strategy is not fixed, however, one might expect that the optimal value (weakly) decreases when a link is added. Surprisingly, the optimal communication strategy might in fact have a higher value after adding a link, so that the sender benefits from a denser network. This is illustrated by means of an extended example.

Example 6.1. Let $|N|=4, \lambda^{0}(X)=1 / 3$, and $k=2$. Consider the following set of networks starting with $g$, obtained by adding a single link: $g_{1}$ and $g_{2}$ from $g$; the line $g_{3}$ from either $g_{1}$ or $g_{2}$; the star $g_{4}$ from $g_{2}$. 

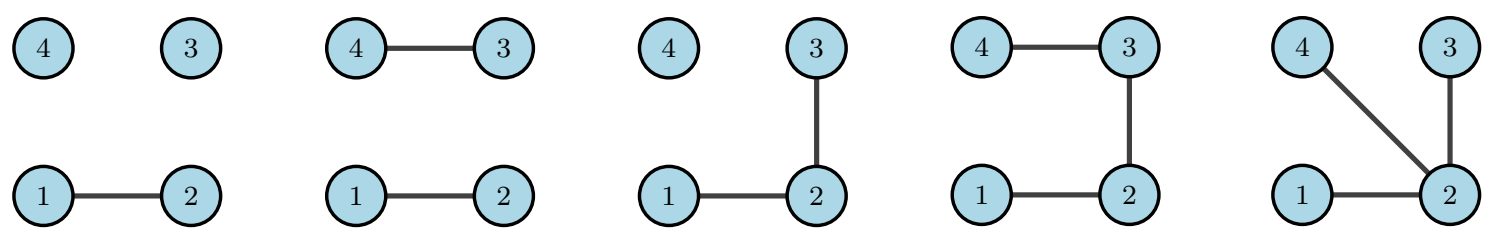

$g: V_{2}^{\pi}(g)=1 \quad g_{1}: V_{2}^{\pi}\left(g_{1}\right)=1 \quad$ Network $g_{2} \quad g_{3}: V_{2}^{\pi^{\prime}}\left(g_{3}\right)=1 \quad g_{4}: V_{2}^{\pi^{\prime \prime}}\left(g_{4}\right)=5 / 6$

Consider the maximum values which can be achieved in each case.

\begin{tabular}{|c|c|c|c|c|c|}
\hline$\pi$ & $\omega=X$ & $\omega=Y$ & $\pi^{\prime}$ & $\omega=X$ & $\omega=Y$ \\
\hline$(x, x, x, x)$ & 1 & 0 & $(x, x, x, x)$ & 1 & 0 \\
\hline$(y, y, x, x)$ & 0 & $\frac{1}{2}$ & $(y, x, x, x)$ & 0 & $\frac{1}{2}$ \\
\hline$(x, x, y, y)$ & 0 & $\frac{1}{2}$ & $(x, x, x, y)$ & 0 & $\frac{1}{2}$ \\
\hline
\end{tabular}

Using communication strategy $\pi$ for $g$ and $g_{1}$ and $\pi^{\prime}$ for $g_{3}$, produces support:

$$
\operatorname{supp}\left(\sigma^{g}\right)=\left\{\left(\frac{1}{2}, \frac{1}{2}, \frac{1}{2}, \frac{1}{2}\right),\left(\frac{1}{2}, \frac{1}{2}, 0,0\right),\left(0,0, \frac{1}{2}, \frac{1}{2}\right)\right\} .
$$

and therefore the maximum possible value of 1 in these cases. ${ }^{6}$ In the case of $g_{4}$, it follows from Proposition 5.2 that the optimal value is $V_{2}^{3}=5 / 6$.

Adding a link between 1 and 4 in $g_{3}$ results in the circle network (call it $g_{5}$ ) analyzed in Example 5.8 with value 1, so starting from $g_{0}$ and adding links in the following way: $g_{0} \rightarrow g \rightarrow g_{1} \rightarrow g_{3} \rightarrow g_{5}$ keeps the optimal value at 1 .

Considering a different sequence like $g \rightarrow g_{2} \rightarrow g_{4}$, adding a link decreases the optimal value. This is not surprising given that the value of the optimal communication strategy with private messages on the empty network dominates (weakly for $n=k$, strongly for $n>k$ ) the value of the public communication strategy (i.e. on the complete network).

What is left to see is if adding a link can strictly increase the maximum value.

Claim 6.2. For any $\hat{\pi} \in \Pi$, it holds that $V_{2}^{\hat{\pi}}\left(g_{2}\right)<1$.

The proof can be found in the Appendix. Claim 6.2 implies that adding a link between $g$ and $g_{2}$ strictly decreases the value of the optimal communication strategy, but adding a link between $g_{2}$ and $g_{3}$ strictly increases it. The sequence $g \rightarrow g_{2} \rightarrow g_{3}$ shows that the sender's value may be higher in a denser network.

\section{Discussion and Conclusion}

\section{Equilibrium}

It is natural to ask whether the optimal communication strategies under sincere voting form an equilibrium. As per Kerman et al. (2020), if in a network with $n$ nodes and quota

\footnotetext{
${ }^{6}$ Using Proposition 4.4, $\pi$ treats identically pairs of nodes which observe the same information within a signal ( 1 and 2, 3 and 4 for $g_{1} ; 1$ and 2 for $g$ ). Additionally, $g_{3}$ is a network which fulfils the conditions of Proposition 5.10 as it is possible to cover precisely $k$ out of the $n$ nodes in every non-anchor signal.
} 
$k$ a communication strategy achieves the optimal value as under the empty network, $V_{k}^{n}$, then the strategy does not form a Bayesian Nash equilibrium (BNE) under sincere voting. This is because in optimal strategies agents are never pivotal in state $X$ and are pivotal with positive probability in state $Y$. So, if all other agents vote sincerely, an agent has an incentive to vote against his belief whenever he considers the true state to be $X$ : as he is not pivotal in state $X$, voting against his belief does not change the outcome of the vote if the true state is indeed $X$, while if the true state is $Y$ he is pivotal and should vote against his belief. This exemplifies the swing voter's curse.

One remedy to the swing voter's curse is never making agents pivotal by having at least $k+1$ agents vote for $x$ in state $Y$ (Kerman et al., 2020). A setting where an optimal communication strategy yields a BNE under sincere voting is given in $g_{3}$ in Example 5.7. By Proposition 4.4, if $k=6$ an optimal communication strategy prescribes making all agents vote for $x$ in state $X$ and 8 (not 6 ) out of 12 agents vote for $x$ in state $Y$. Hence, no agent is ever pivotal and sincere voting is a BNE. Similarly, the optimal communication strategy in a complete network is public and yields a BNE under sincere voting.

\section{Concluding Remarks}

This paper investigates the optimal persuasion of voters who exchange private information with each other. This is modeled as a fixed network where direct neighbors can perfectly observe each other's private messages sent by a centralized body. The sender wants to implement a certain proposal and commits in advance to a communication strategy which sends correlated messages to the receivers. This presents several difficulties as most of the assumptions that hold under standard multiple-receiver Bayesian persuasion models fail. Crucially, while there are parallels to the empty network model, straightforward or anonymous strategies are not generally optimal, and neither are strategies which are truth-telling in state $X$.

The paper tests the naive intuition that more information provided to the receivers through the network would make them less manipulable. This is true in some cases. However, the presence of a network structure does not always impede the persuasion abilities of the sender. In fact, the value of the optimal communication strategy is not monotone in terms of network density. Several network structures are identified where the sender can achieve the optimal value with private messages as under the empty network and their corresponding optimal strategies are outlined. While many of these situations rely on some form of symmetry or regularity of the network, this can also be achieved on general network structures, e.g. in networks with sufficiently many isolated nodes.

\section{A Proofs}

Proof of Lemma 3.5. Let $\pi \in \Pi$ be such that $V_{k}^{\pi}(g)>0$. Assume to the contrary that $A n(\pi)=\emptyset$. Then for every $s^{\prime} \in S^{\pi}$ it holds that $\pi\left(s^{\prime} \mid X\right) \lambda^{0}(X)<\pi\left(s^{\prime} \mid Y\right) \lambda^{0}(Y)$. Since 
$V_{k}^{\pi}(g)>0$, there exists $i \in N$ and $s \in S^{\pi}$ such that $\alpha_{i}^{\pi, g}\left(s_{i}(g)\right)=x$. That is,

$$
\lambda_{i}^{s, g}(X)=\frac{\sum_{t \in A_{i}^{\pi}(g, s)} \pi(t \mid X) \lambda^{0}(X)}{\sum_{t \in A_{i}^{\pi}(g, s)} \pi(t \mid X) \lambda^{0}(X)+\sum_{t \in A_{i}^{\pi}(g, s)} \pi(t \mid Y) \lambda^{0}(Y)} \geq \frac{1}{2} .
$$

Rearranging the terms in (4), we obtain:

$$
\sum_{t \in A_{i}^{\pi}(g, s)} \pi(t \mid X) \lambda^{0}(X) \geq \sum_{t \in A_{i}^{\pi}(g, s)} \pi(t \mid Y) \lambda^{0}(Y)
$$

a contradiction, since $\pi\left(s^{\prime} \mid X\right) \lambda^{0}(X)<\pi\left(s^{\prime} \mid Y\right) \lambda^{0}(Y)$ for every $s^{\prime} \in S^{\pi}$.

Notice that this observation implies that every $x$ vote under a strategy with $V_{k}^{\pi}>0$ must be associated with at least one anchor.

Proof of Lemma 3.7. Fix $i \in N$ and let $\left|S_{i}^{\pi}\right| \geq 2$. Note that if $\alpha_{i}^{\pi, g}\left(s_{i}(g)\right)=x$ for some $s \in S^{\pi}$, then $s \in \cup_{t \in A n(\pi)} A^{\pi}(g, t)$, that is, whenever $i$ votes in favor of $x$, his observation is associated with some anchor(s). Moreover, for each $t \in A n(\pi)$ and $s, s^{\prime} \in A_{i}^{\pi}(g, t)$ it holds that $s_{i}=s_{i}^{\prime}=t_{i}$. Note that if $s^{\prime \prime} \notin \cup_{t \in A n(\pi)} A_{i}^{\pi}(g, t)$, then $\alpha_{i}^{\pi, g}\left(s_{i}^{\prime \prime}(g)\right) \neq x$, that is, whenever a receiver observes a private message not associated with any anchor, he does not vote in favor of $x$ and neither do any of his neighbors. That is, for any $j \in N_{i}(g)$ it holds that $\alpha_{j}^{\pi, g}\left(s_{j}^{\prime \prime}(g)\right) \neq x$.

Define $\pi^{\prime}: \Omega \rightarrow \Delta(\Omega)^{n}$ such that in $\pi$ we replace all messages $s_{i} \in S_{i}^{\pi}$ such that $s \notin \cup_{t \in A n(\pi)} A_{i}^{\pi}(g, t)$ with a message $m_{i}^{y} \in S_{i} \backslash S_{i}^{\pi}$. It follows that $A n\left(\pi^{\prime}\right)=A n(\pi)$ and $i$ observes at most $\left|A n\left(\pi^{\prime}\right)\right|+1$ messages under $\pi^{\prime}$, i.e. $\left|S_{i}^{\pi^{\prime}}\right| \leq\left|A n\left(\pi^{\prime}\right)\right|+1$. Let $s \in S^{\pi}$ be such that $s \notin \cup_{t \in A n(\pi)} A_{i}^{\pi}(g, t)$ and therefore, $\alpha_{i}^{\pi, g}\left(s_{i}(g)\right)=y$. If $s^{\prime} \in S^{\pi^{\prime}}$ is obtained from $s \in S^{\pi}$ via replacing $s_{i}$ with $m_{i}^{y}$, then $\alpha_{i}^{\pi^{\prime}, g}\left(s_{i}^{\prime}(g)\right)=x$ if and only if $\alpha_{i}^{\pi, g}\left(s_{i}(g)\right)=x$. That is, the number of $x$ votes are the same under $s$ and $s^{\prime}$. Thus, $V_{k}^{\pi^{\prime}}(g)=V_{k}^{\pi}(g)$.

Proof of Proposition 4.1. Let $\pi \in \Pi$. For each $i \in N$, assume that $\left|S_{i}^{\pi}(g)\right|=c(i)$. Let $R(i)=\left\{m_{i}^{1}, \ldots, m_{i}^{c(i)}\right\} \subseteq S_{i}$ be a set of distinct messages for $i$. Moreover for any $j \in N$, $q \in\{1, \ldots, c(i)\}$, and $q^{\prime} \in\{1, \ldots, c(j)\}$ let $m_{i}^{q} \neq m_{j}^{q^{\prime}}$.

For each $i \in N$, let $\phi_{i}: S_{i}^{\pi}(g) \rightarrow R(i)$ be a bijection, so each information neighborhood of $i$ is mapped to a unique message in $R(i)$. For each $\omega \in \Omega$ and $s^{\prime} \in S$, define $\pi^{\prime} \in \Pi$ :

$$
\pi^{\prime}\left(s^{\prime} \mid \omega\right)= \begin{cases}\pi(s \mid \omega) & \text { if } \phi_{i}\left(s_{i}(g)\right)=s_{i}^{\prime}, \quad \forall i \in N \\ 0 & \text { otherwise. }\end{cases}
$$

Note that the definition of $\pi^{\prime}$ implies that there is a bijection $\phi: S^{\pi} \rightarrow S^{\pi^{\prime}}$ such that for each $i \in N, \phi(s)=s^{\prime}$ if and only if $\phi_{i}\left(s_{i}(g)\right)=s_{i}^{\prime}$. Hence, $\pi^{\prime}$ is a communication strategy.

We want to show that the value of $\pi^{\prime}$ under the empty network is equal to the value of $\pi$ under $g$, i.e., $V_{k}^{\pi^{\prime}}\left(g_{0}\right)=V_{k}^{\pi}(g)$. What remains to be shown is that each receiver $i$ has the same posterior belief upon observing $s_{i}(g)$ under $\pi$ and upon observing $\phi_{i}\left(s_{i}(g)\right)$ under $\pi^{\prime}$. Let $s^{\prime} \in S^{\pi^{\prime}}$ be such that $s_{i}^{\prime} \in\left\{m_{i}^{1}, \ldots, m_{i}^{c(i)}\right\}$. For any $\omega \in \Omega$, we have 


$$
\begin{aligned}
\lambda_{i}^{s^{\prime}}(\omega) & =\frac{\sum_{s \in S^{\prime}: s_{i}=s_{i}^{\prime}} \pi^{\prime}(s \mid \omega) \lambda^{0}(\omega)}{\sum_{\omega^{\prime} \in \Omega} \sum_{s \in S^{\pi^{\prime}}: s_{i}=s_{i}^{\prime}} \pi^{\prime}\left(s \mid \omega^{\prime}\right) \lambda^{0}\left(\omega^{\prime}\right)}=\frac{\sum_{s \in S^{\pi}: s_{i}(g)=\phi^{-1}\left(s_{i}^{\prime}\right)} \pi(s \mid \omega) \lambda^{0}(\omega)}{\sum_{\omega^{\prime} \in \Omega} \sum_{s \in S^{\pi}: s_{i}(g)=\phi^{-1}\left(s_{i}^{\prime}\right)} \pi^{\prime}\left(s \mid \omega^{\prime}\right) \lambda^{0}\left(\omega^{\prime}\right)} \\
& =\frac{\sum_{s \in A_{i}^{\pi}\left(g, \phi^{-1}\left(s^{\prime}\right)\right)} \pi(s \mid \omega) \lambda^{0}(\omega)}{\sum_{\omega^{\prime} \in \Omega} \sum_{s \in A_{i}^{\pi}\left(g, \phi^{-1}\left(s^{\prime}\right)\right)} \pi\left(s \mid \omega^{\prime}\right) \lambda^{0}\left(\omega^{\prime}\right)}=\lambda_{i}^{\phi^{-1}\left(s^{\prime}\right), g}(\omega) .
\end{aligned}
$$

Thus, for each $s \in S^{\pi}$ it holds that $\alpha^{\pi, g}(s)=\alpha^{\pi^{\prime}, g_{0}}(\phi(s))$. Hence, $V_{k}^{\pi^{\prime}}\left(g_{0}\right)=V_{k}^{\pi}(g)$. Since any $\pi \in \Pi$ on some network $g$ can be replicated on the empty network, $V_{k}^{n} \geq V_{k}^{\pi}(g)$.

Proof of Proposition 4.3. Assume that $\lambda^{0}(Y) / \lambda^{0}(X)=\ell,\left|\left\{i \in N: \delta_{i}^{g}=0\right\}\right|=q \geq k$ and $2 k \geq n$. So, there are $q$ singleton receivers and $n-q$ connected receivers. Denote the set of singleton receivers by $N^{q}$ and the set of connected receivers by $N^{c}$.

Let $S^{\prime}=\{x, y\}^{n}$. Define:

$$
R=\left\{s \in S^{\prime}: \forall i \in N^{c}, s_{i}=x \text { and }\left|\left\{j \in N^{q}: s_{j}=x\right\}\right|=k-(n-q)\right\} .
$$

In words, $R$ is the set of signals in which all connected receivers and $k-n+q$ of the singleton receivers observe $x$. Note that $k-(n-q)$ is the required amount of $x$ votes to fulfil the quota given that all connected receivers vote for $x$. Moreover, $|R|=\left(\begin{array}{c}q \\ k-n+q\end{array}\right)$.

Finally, define:

$$
T=\left\{t \in S^{\prime}: \forall i \in N^{c}, t_{i}=y \text { and }\left|\left\{j \in N^{q}: t_{j}=x\right\}\right|=k\right\} .
$$

So, $T$ is the set of signals in which all $n-q$ connected receivers and $q-k$ singleton receivers observe $y$, while $k$ singleton receivers observe $x$. Here $|T|=\left(\begin{array}{l}q \\ k\end{array}\right)$.

Let $\bar{x}$ be such that $\bar{x}_{i}=x$ for all $i \in N$ and define $\bar{y}$ analogously. Define $\pi$ as follows:

$$
\pi(s \mid \omega)= \begin{cases}1 & \text { if } s=\bar{x} \text { and } \omega=X, \\
1-\frac{n}{k \ell} & \text { if } s=\bar{y} \text { and } \omega=Y, \\
\frac{n-k}{q \ell}\left(\begin{array}{c}
q-1 \\
k-1
\end{array}\right)^{-1} & \text { if } s \in T \text { and } \omega=Y, \\
\frac{1}{\left(\begin{array}{c}
q \\
k-n+q
\end{array}\right) \ell} & \text { if } s \in R \text { and } \omega=Y .\end{cases}
$$

It can be easily checked that $\pi$ is a communication strategy:

$$
\begin{aligned}
\sum_{s \in S^{\pi}} \pi(s \mid Y) & =1-\frac{n}{k \ell}+\left(\begin{array}{l}
q \\
k
\end{array}\right) \frac{n-k}{q \ell}\left(\begin{array}{l}
q-1 \\
k-1
\end{array}\right)^{-1}+\left(\begin{array}{c}
q \\
k-n+q
\end{array}\right) \frac{1}{\left(\begin{array}{c}
q \\
k-n+q
\end{array}\right) \ell} \\
& =1-\frac{n}{k \ell}+\frac{n-k}{q \ell} \frac{q}{k}+\frac{1}{\ell}=1-\frac{n}{k \ell}+\frac{n}{k \ell}=1 .
\end{aligned}
$$

We will show that $V_{k}^{\pi}(g)=V_{k}^{n}=\lambda^{0}(X)(n+k) / k$. Under $\pi$, the connected agents always observe the same message. For any $s \in S^{\pi}$ with $s_{i}=x$ for all $i \in N^{c}$, we denote the information neighborhood $s_{i}(g)$ of a connected receiver by $\tilde{x}(i)$. Note that for any $i \in N^{c}$, we have $\pi_{i}(\tilde{x}(i) \mid Y)=\frac{\left(\begin{array}{c}q \\ k-n+q\end{array}\right)}{\left(\begin{array}{c}q \\ k-n+q\end{array}\right) \ell}=1 / \ell$. Hence, for any $i \in N^{c}$ and $s \in S^{\pi}$ with $s_{i}(g)=\tilde{x}(i)$ it holds that: 


$$
\lambda_{i}^{s, g}(X)=\frac{\pi_{i}(\tilde{x}(i) \mid X) \lambda^{0}(X)}{\pi_{i}(\tilde{x}(i) \mid X) \lambda^{0}(X)+\pi_{i}(\tilde{x}(i) \mid Y) \lambda^{0}(Y)}=\frac{\lambda^{0}(X)}{\lambda^{0}(X)+\frac{1}{\ell} \lambda^{0}(Y)}=\frac{1}{2} .
$$

Thus, a connected receiver $i$ votes in favor of $x$ upon observing $\tilde{x}(i)$.

Now, let $i \in N^{q}$. The probability of $i$ observing $x$ in state $Y$ is given by:

$$
\begin{aligned}
\pi_{i}(x \mid Y) & =\sum_{s \in S^{\pi}: s_{i}=x} \pi(s \mid Y)=\sum_{s \in R: s_{i}=x} \pi(s \mid Y)+\sum_{t \in T: t_{i}=x} \pi(t \mid Y) \\
& =\frac{\left(\begin{array}{c}
q-1 \\
k-n+q-1
\end{array}\right)}{\left(\begin{array}{c}
q \\
k-n+q
\end{array}\right) \ell}+\left(\begin{array}{c}
q-1 \\
k-1
\end{array}\right) \frac{n-k}{q \ell}\left(\begin{array}{c}
q-1 \\
k-1
\end{array}\right)^{-1}=\frac{k-n+q}{q \ell}+\frac{n-k}{q \ell}=\frac{1}{\ell} .
\end{aligned}
$$

Similar calculations as in the connected receiver case follow and thus, each singleton receiver has posterior $1 / 2$ that the state is $X$ upon observing $x$. The value of $\pi$ is then:

$$
\begin{aligned}
V_{k}^{\pi}(g) & =\lambda^{0}(X) \cdot 1+\lambda^{0}(Y)\left(\frac{n-k}{k \ell}+\frac{1}{\ell}\right)=\lambda^{0}(X)+\lambda^{0}(Y) \frac{n}{k \ell} \\
& =\lambda^{0}(X)+\lambda^{0}(Y) \frac{n}{k} \frac{\lambda^{0}(X)}{\lambda^{0}(Y)}=\frac{n+k}{k} \lambda^{0}(X)=V_{k}^{n} .
\end{aligned}
$$

Proof of Proposition 4.4. First, note that since $\bar{N}_{i}(g)=\bar{N}_{j}(g)$, we have $A_{i}^{\pi}(g, s)=$ $A_{j}^{\pi}(g, s)$. Hence, $i$ and $j$ have the same posterior belief, i.e. for any $\omega \in \Omega$ and any $s \in S^{\pi}$ :

$$
\lambda_{i}^{s, g}(\omega)=\frac{\sum_{t \in A_{i}^{\pi}(g, s)} \pi(t \mid \omega) \lambda^{0}(\omega)}{\sum_{\omega^{\prime} \in \Omega} \sum_{t \in A_{i}^{\pi}(g, s)} \pi\left(t \mid \omega^{\prime}\right) \lambda^{0}\left(\omega^{\prime}\right)}=\frac{\sum_{t \in A_{j}^{\pi}(g, s)} \pi(t \mid \omega) \lambda^{0}(\omega)}{\sum_{\omega^{\prime} \in \Omega} \sum_{t \in A_{j}^{\pi}(g, s)} \pi\left(t \mid \omega^{\prime}\right) \lambda^{0}\left(\omega^{\prime}\right)}=\lambda_{j}^{s, g}(\omega) .
$$

Let $\left|S_{i}^{\pi} \times S_{j}^{\pi}\right|=c$. Let $R=\left\{m^{1}, \ldots, m^{c}\right\}$ be a set of distinct messages. Define a bijection $\phi: S_{i}^{\pi} \times S_{j}^{\pi} \rightarrow R$. That is, for any tuple $\left(s_{i}, s_{j}\right),\left(t_{i}, t_{j}\right) \in S_{i}^{\pi} \times S_{j}^{\pi}$ it holds that $\phi\left(s_{i}, s_{j}\right)=$ $\phi\left(t_{i}, t_{j}\right)$ if and only if $\left(s_{i}, s_{j}\right)=\left(t_{i}, t_{j}\right)$, so that each distinct combination of messages of $i$ and $j$ (and not every distinct neighborhood) is mapped to a distinct message in $R$.

Define $S^{\prime}=\left\{s^{\prime} \in S \mid s \in S^{\pi}, s_{-i j}^{\prime}=s_{-i j}\right.$ and $\left.\phi\left(s_{i}, s_{j}\right)=s_{i}^{\prime}=s_{j}^{\prime} \in R\right\}$. In words, $S^{\prime}$ consists of signals obtained by replacing the messages of $i$ and $j$ with distinct messages in $R$ (for each distinct message combination) and leaving the other receivers' messages unchanged, in each signal in $S^{\pi}$. Let $\tau: S^{\pi} \rightarrow S^{\prime}$ be a bijection such that for any $s \in S^{\pi}$ we have $\tau(s)=s^{\prime}$ if $\tau\left(s_{i}, s_{j}\right)=s_{i}^{\prime}=s_{j}^{\prime}$ and $s_{-i j}^{\prime}=s_{-i j}$.

For every $s \in S^{\pi}$ and $\omega \in \Omega$, define $\pi^{\prime}(\tau(s) \mid \omega)=\pi(s \mid \omega)$. It is clear that $\pi^{\prime}$ is a communication strategy. Note that since the probability weights are the same under $\pi$ and $\pi^{\prime}$, receivers $i$ and $j$ still have the same posterior belief under $\pi^{\prime}$, i.e. for any $\omega \in \Omega$ and $s \in S^{\pi^{\prime}}$ it holds that $\lambda_{i}^{s, g}(\omega)=\lambda_{j}^{s, g}(\omega)$.

Next, we show that for any $r \in \bar{N}_{i}(g), \omega \in \Omega$, and $s \in S^{\pi}$ we have $\lambda_{r}^{s, g}(\omega)=\lambda_{r}^{\tau(s), g}(\omega)$.

$$
\begin{aligned}
\lambda_{r}^{s, g}(\omega) & =\frac{\sum_{t \in A_{r}^{\pi}(g, s)} \pi(t \mid \omega) \lambda^{0}(\omega)}{\sum_{\omega^{\prime} \in \Omega} \sum_{t \in A_{r}^{\pi}(g, s)} \pi\left(t \mid \omega^{\prime}\right) \lambda^{0}\left(\omega^{\prime}\right)}=\frac{\sum_{t \in A_{r}^{\pi}(g, s)} \pi^{\prime}(\tau(t) \mid \omega) \lambda^{0}(\omega)}{\sum_{\omega^{\prime} \in \Omega} \sum_{t \in A_{r}^{\pi}(g, s)} \pi^{\prime}\left(\tau(t) \mid \omega^{\prime}\right) \lambda^{0}\left(\omega^{\prime}\right)} \\
& =\frac{\sum_{t^{\prime} \in A_{r}^{\pi^{\prime}(g, \tau(s))}} \pi^{\prime}\left(t^{\prime} \mid \omega\right) \lambda^{0}(\omega)}{\sum_{\omega^{\prime} \in \Omega} \sum_{t^{\prime} \in A_{r}^{\pi^{\prime}}(g, \tau(s))} \pi^{\prime}\left(t^{\prime} \mid \omega^{\prime}\right) \lambda^{0}\left(\omega^{\prime}\right)}=\lambda_{r}^{\tau(s), g}(\omega) .
\end{aligned}
$$


Finally, any $r \notin \bar{N}_{i}(g)$ has the same posterior belief under $\pi$ and $\pi^{\prime}$, as it is not affected by the transformation. Hence, $V_{k}^{\pi^{\prime}}(g)=V_{k}^{\pi}(g)$.

Proof of Proposition 4.7. As all components are complete, all of their elements can be sent the same private message within every signal by Proposition 4.4. Let $q \in \mathbb{N}$ be such that for each $\mathcal{C}^{\prime} \in \mathfrak{C}^{q}$ it holds that $\sum_{C \in \mathcal{C}^{\prime}}|C| \geq k$. Note that there are $\left(\begin{array}{l}\ell \\ q\end{array}\right)$ many ways to choose $q$ components such that the total number of receivers in the components is at least $k$. Then, by Theorem 3.1 it follows that there exists $\pi \in \Pi$ such that $V_{k}^{\pi}(g)=V_{q}^{\ell}$.

Proof of Proposition 5.2. First, Lemma A.1 shows that without loss of generality the center node is not pivotal whenever it votes for $x$. Denote the center node in $g$ by $c \in N$.

Lemma A.1. Let $g \in G(N)$ be a star, $k<n$, and let $\pi \in \Pi$ be a communication strategy such that there exists $s \in S^{\pi}$ with $\alpha_{c}^{\pi, g}(s)=x$ and $c$ is pivotal in $s$. Then there exists $\pi^{\prime} \in \Pi$ such that for any $s^{\prime} \in S^{\pi^{\prime}}$ with $\alpha_{c}^{\pi^{\prime}, g}\left(s^{\prime}\right)=x$, the center node is not pivotal in $s^{\prime}$ and $V_{k}^{\pi^{\prime}}(g)=V_{k}^{\pi}(g)$.

Proof of Lemma A.1. Note that $t_{c}(g)=t$ for all $t \in S^{\pi}$ (the information neighbourhood of $c$ is the whole $g$ ). Therefore, $\lambda_{c}^{s, g}(X) \geq 1 / 2$ if and only if $s$ is an anchor, so if $\alpha_{c}^{\pi, g}(s)=x$ for some $s \in S^{\pi}$, it follows that $s \in A n(\pi)$. Moreover, if $c$ is pivotal in this $s$ and $k<n$, there exists a peripheral $i \in N$ (i.e. $i \neq c$ ) such that $\alpha_{i}^{\pi, g}\left(s_{i}(g)\right)=y$. Furthermore, $i$ votes for $y$ in any other signal he associates with $s$, i.e. for any $t \in A_{i}^{\pi}(g, s)$ it holds that $\alpha_{i}^{\pi, g}\left(t_{i}(g)\right)=y$. Thus, replacing $i$ 's message in the anchor $s$ with a unique message would enable $i$ to uniquely identify the anchor and hence reverse $i$ 's vote from $y$ to $x$ in $s$ (see the remark after Definition 3.4). Since $c$ votes for $x$ if and only if the signal is an anchor, $c$ 's vote would not change if the probabilities of the communication strategy do not change. It would also keep everyone else's vote the same, as $i$ is only observed by $c$.

To this end, let $S^{\prime} \subseteq S^{\pi}$ such that $S^{\prime}=\left\{s \in S^{\pi} \mid \alpha_{c}^{\pi, g}(s)=x\right.$ and $c$ is pivotal in $\left.s\right\}$. In particular, let $S^{\prime}=\left\{s^{1}, \ldots, s^{r}\right\}$. Fix $i \in N$ such that for some $t \in S^{\prime}$ it holds that $\alpha_{i}^{\pi, g}(t)=y$ and notice that such $i$ exists as per the discussion above. Let $R=\left\{m^{1}, \ldots, m^{r}\right\}$ be a set of distinct messages such that for any $j \in\{1, \ldots, r\}, m^{j} \notin S_{i}^{\pi}$. Let $S^{\prime \prime} \subseteq S$ and define a bijection $\phi: S^{\prime} \rightarrow S^{\prime \prime}$ such that for every $j \in\{1, \ldots, r\}$ and $s^{j} \in S^{\prime}$ it holds that $\phi_{i}\left(s_{i}^{j}\right)=m^{j}$ and $\phi_{-i}\left(s_{-i}^{j}\right)=s_{-i}^{j}$.

Now, for any $\omega \in \Omega$ and any $s^{\prime} \in\left(S^{\pi} \backslash S^{\prime}\right) \cup S^{\prime \prime}$, let $\pi^{\prime} \in \Pi$ be defined by:

$$
\pi^{\prime}\left(s^{\prime} \mid \omega\right)= \begin{cases}\pi\left(\phi^{-1}\left(s^{\prime}\right) \mid \omega\right) & \text { if } s^{\prime} \in S^{\prime \prime} \\ \pi\left(s^{\prime} \mid \omega\right) & \text { if } s^{\prime} \in S^{\pi} \backslash S^{\prime} .\end{cases}
$$

That is, $S^{\pi^{\prime}}=\left(S^{\pi} \backslash S^{\prime}\right) \cup S^{\prime \prime}$. By the definition of an anchor, for any $t \in S^{\prime \prime}$, we have $\alpha_{i}^{\pi^{\prime}, g}\left(t_{i}(g)\right)=x$, since by construction $i$ observes the unique message only in this anchor $t$. Therefore, there are $k+1$ receivers voting for $x$ in $t$, which implies that the center node is no longer pivotal. Since $\pi^{\prime}$ preserves all probability weights it is true that if $s \in S^{\prime \prime}$, then $s \in A n\left(\pi^{\prime}\right)$. 
Moreover, $i$ 's votes in signals that are not in $S^{\prime \prime}$ are unchanged, i.e. for any $t \in S^{\pi^{\prime}} \backslash S^{\prime \prime}$, $\alpha_{i}^{\pi, g}\left(t_{i}(g)\right)=\alpha_{i}^{\pi^{\prime}, g}\left(t_{i}(g)\right)$. This holds because if $s \in S^{\prime}$ and $t \in A_{i}^{\pi}(g, s)$, then it holds that $\alpha_{i}^{\pi, g}\left(t_{i}(g)\right)=y$ by the definition of $S^{\prime}$ and the selection of $i$. The transformation removes the anchors in $S^{\prime \prime}$ from the association set of every signal $t \in S^{\pi^{\prime}} \backslash S^{\prime \prime}$, so if $s \in S^{\prime \prime}$ then for every $t \in S^{\pi^{\prime}} \backslash S^{\prime \prime}$ it is true that $t \notin A_{i}^{\pi^{\prime}}(g, s)$. This makes it even less likely that $i$ would vote for $x$ in such signals, preserving its $y$ votes in them between $\pi$ and $\pi^{\prime}$. The transformation does not affect any other receivers' votes, hence $V_{k}^{\pi^{\prime}}(g)=V_{k}^{\pi}(g)$.

By Lemma A.1, assume without loss of generality that under $\pi$, the center node is not pivotal in signals in which he votes for $x$.

For all nodes $i \in N$ and all $t \notin A n(\pi)$, if $t_{c} \neq s_{c}$ for all $s \in A n(\pi)$ then $\lambda_{i}^{t, g}(X)<1 / 2$. So, if in a certain signal the center of a star network receives a message different from all anchors, all receivers would vote $y$ in this signal.

Note that for two anchors $s, t \in A n(\pi)$ with $s_{c} \neq t_{c}$, it holds that $A^{\pi}(g, s) \cap A^{\pi}(g, t)=\emptyset$. Define a bijection $\phi: S^{\pi} \rightarrow S^{\prime}$ such that $\phi(s)=s^{\prime}$ if $s_{c}^{\prime}=x$ and for every $j \in N \backslash\{c\}$, $s_{j}^{\prime}=\left(s_{j}, s_{c}\right)$. That is, in signals in $S^{\prime}$ the center node always observes $x$ and the periphery nodes' messages are modified so that they contain the information previously provided by the center in signal $s$. In other words, the information that the center reveals to the periphery nodes is shifted to them while the center observes the same message $x$ in every signal.

For every $s^{\prime} \in S^{\prime}$ such that $\phi(s)=s^{\prime}$ and $\omega \in \Omega$, let $\pi^{\prime} \in \Pi$ be defined by $\pi^{\prime}\left(s^{\prime} \mid \omega\right)=$ $\pi\left(\phi^{-1}\left(s^{\prime}\right) \mid \omega\right)$. As the probabilities of corresponding signals are the same under $\pi^{\prime}$ as under $\pi$ and the center's information under $\pi$ is shifted to the periphery nodes under $\pi^{\prime}$ (which are observed by the center), the center node's vote does not change. Moreover, the votes of the periphery nodes do not change either. To see this, note that for any $t^{\prime} \in A_{i}^{\pi^{\prime}}\left(g, s^{\prime}\right)$ there exists $t \in A_{i}^{\pi}(g, s)$ such that $\phi(t)=t^{\prime}$. This, together with the definition of $\phi$ implies that $\sum_{t^{\prime} \in A_{i}^{\pi^{\prime}}\left(g, s^{\prime}\right)} \pi^{\prime}\left(t^{\prime} \mid \omega\right)=\sum_{t \in A_{i}^{\pi}(g, s)} \pi(t \mid \omega)$. Thus, each periphery node has the same posterior belief upon observing $s \in S^{\pi}$ and $\phi(s) \in S^{\pi^{\prime}}$. Hence, $V_{k}^{\pi^{\prime}}(g)=V_{k}^{\pi}(g)$.

As the center node always observes the same message under $\pi^{\prime}$, it has no effect on the voting decisions of the other receivers. Moreover, the center node is never pivotal in signals in which he votes for $x$. Observe that under $\pi^{\prime}$, it is as if the center is always voting for $y$, since all of his $y$ votes are preserved in $\pi^{\prime}$ and none of his $x$ votes have an impact on whether a signal implements $x$ or not. Thus, the setup is equivalent to having an empty network with $n-1$ nodes. Hence, we can assume without loss of generality that there exists a communication strategy $\pi^{\prime \prime} \in \Pi$ with $\left|S_{i}^{\pi^{\prime \prime}}\right|=2$ for any $i \in N$ such that $V_{k}^{n-1}=V_{k}^{\pi^{\prime \prime}}(g) \geq V_{k}^{\pi^{\prime}}(g)$.

Proof of Proposition 5.4. Take the following communication strategy $\pi$ : 


\begin{tabular}{|c|c|c|}
\hline$\pi$ & $\omega=X$ & $\omega=Y$ \\
\hline$(x, x, x, x, \ldots, x, x, x, x)$ & 1 & 0 \\
\hline$(\underbrace{y, \ldots, y}, x, x, x, \ldots, x, x)$ & 0 & $\frac{w_{1}}{n}$ \\
\hline 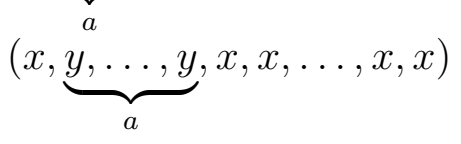 & 0 & $\frac{w_{1}}{n}$ \\
\hline & $\ldots$ & $\cdots$ \\
\hline$(x, x, \ldots, x, x, \underbrace{y, \ldots, y}, x)$ & 0 & $\frac{w_{1}}{n}$ \\
\hline$(x, x, \ldots, x, x, x, \underbrace{y, \ldots, y}_{a})$ & 0 & $\frac{w_{1}}{n}$ \\
\hline$\cdots$ & $\cdots$ & $\cdots$ \\
\hline$(\underbrace{y, \ldots, y}_{a-1}, x, x, \ldots, x, x, y)$ & 0 & $\frac{w_{1}}{n}$ \\
\hline$(y, y, y, y, \ldots, y, y, y, y)$ & 0 & $w_{2}$ \\
\hline
\end{tabular}

with $w_{1}=r_{k}^{*}, w_{2}=r_{0}^{*}$ from Proposition 3.1 and $a=n-2-k$. This makes a total of $n+2$ signals. Every node observes a message $y$ in their information neighborhood in exactly $a+3$ signals. This leaves $n-1-a$ signals in which $i$ and all neighbors of $i$ observe $x$. Given $s^{\prime} \in S^{\pi}$, denote the information neighborhood $s_{i}^{\prime}(g)$ of $i \in N$ by $\tilde{x}(i)$ if for all $j \in \bar{N}_{i}(g)$ it holds that $s_{j}^{\prime}=x$. Let $i \in N$ and $s \in S^{\pi}$ be such that $s_{i}(g)=\tilde{x}(i)$. It holds that:

$$
\lambda_{i}^{s, g}(X)=\frac{\sum_{t \in A_{i}^{\pi}(g, s)} \pi(t \mid X) \lambda^{0}(X)}{\sum_{t \in A_{i}^{\pi}(g, s)} \pi(t \mid X) \lambda^{0}(X)+\sum_{t \in A_{i}^{\pi}(g, s)} \pi(t \mid Y) \lambda^{0}(Y)}=\frac{\lambda^{0}(X)}{\lambda^{0}(X)+\frac{(n-2-a) w_{1}}{n} \lambda^{0}(Y)} .
$$

Therefore,

$$
\lambda_{i}^{s, g}(X)= \begin{cases}\frac{\lambda^{0}(X)}{1 \lambda^{0}(X)+\frac{(n-2-a)}{n} \frac{\lambda^{0}(X) n}{\lambda^{0}(Y) \lambda^{0}(Y)}=\frac{1}{1+\frac{n-2-a}{k}}=1 / 2} & \text { if } \lambda^{0}(X)<\frac{k}{k+n}, \\ \frac{\lambda^{0}(X)}{\lambda^{0}(X)+(n-2-a) \lambda^{0}(Y) / n} \geq 1 / 2 & \text { if } \lambda^{0}(X) \geq \frac{k}{k+n},\end{cases}
$$

as the second condition always holds for $\lambda^{0}(X) \geq \frac{k}{k+n}$.

In each signal $s \in S^{\pi}$ such that there exists $i \in N$ with $s_{i}=y$, there are $n-2-a$ many receivers $j \in N$ such that $s_{j}(g)=\tilde{x}(j)$. Therefore, all these signals persuade at least $k$ agents, i.e. for such $s \in S^{\pi}$ we have $\left|\left\{i \in N: \alpha_{i}^{\pi, g}\left(s_{i}(g)\right)=x\right\}\right| \geq k$. The value is equal to the empty network value, i.e. $V_{k}^{\pi}(g)=\lambda^{0}(X) \cdot 1+\lambda^{0}(Y) w_{1}=\min \left\{\frac{n+k}{k}, 1\right\}=V_{k}^{n}$.

Proof of Proposition 5.6. Let $S^{\prime}=\{x, y\}^{n}$. Let $T$ be the set of signals in $S^{\prime}$ in which exactly one receiver observes $y$, i.e. $T=\left\{s \in S^{\prime} \mid \exists i \in N: s_{i}=y\right.$ and $\left.s_{-i}=x\right\}$. Let $\bar{x} \in S$ 
be such that $\bar{x}_{i}=x$ for all $i \in N$ and define $\bar{y}$ analogously. Define $\pi \in \Pi$ :

$$
\pi(s \mid \omega)= \begin{cases}1 & \text { if } s=\bar{x} \text { and } \omega=X \\ \frac{w_{1}}{n} & \text { if } s \in T \text { and } \omega=Y \\ w_{2} & \text { if } s=\bar{y} \text { and } \omega=Y \\ 0 & \text { otherwise }\end{cases}
$$

with $w_{1}=r_{k}^{*}, w_{2}=r_{0}^{*}$ from Theorem 3.1. This makes a total of $n+2$ signals. Since the network is regular, every node observes message $y$ in their information neighborhood in exactly $\delta+2$ signals. This leaves $n-\delta$ signals where an agent $i \in N$ observes $\tilde{x}(i)$ (in particular, there are $n-\delta-1$ such signals in state $Y$ ). Let $i \in N$ and $s \in S^{\pi}$ be such that $s_{i}(g)=\tilde{x}(i)$. It holds that:

$$
\lambda_{i}^{s, g}(X)=\frac{\sum_{t \in A_{i}^{\pi}(g, s)} \pi(t \mid X) \lambda^{0}(X)}{\sum_{t \in A_{i}^{\pi}(g, s)} \pi(t \mid X) \lambda^{0}(X)+\sum_{t \in A_{i}^{\pi}(g, s)} \pi(t \mid Y) \lambda^{0}(Y)}=\frac{\lambda^{0}(X)}{\lambda^{0}(X)+\frac{(n-\delta-1) w_{1}}{n} \lambda^{0}(Y)} .
$$

Therefore,

$$
\lambda_{i}^{s, g}(X)= \begin{cases}\frac{1 \lambda^{0}(X)}{1 \lambda^{0}(X)+\frac{(n-\delta-1)}{n} \frac{\lambda^{0}(X) n}{\lambda^{0}(Y) k} \lambda^{0}(Y)}=\frac{1}{1+\frac{n-\delta-1}{k}}=\frac{1}{2} & \text { if } \lambda^{0}(X)<\frac{k}{k+n} \\ \frac{\lambda^{0}(X)}{\lambda^{0}(X)+\frac{k}{n} \lambda^{0}(Y)} \geq 1 / 2 & \text { if } \lambda^{0}(X) \geq \frac{k}{k+n}\end{cases}
$$

as the second condition always holds for for $\lambda^{0}(X) \geq \frac{k}{k+n}$.

In each signal $s \in S^{\pi}$ such that there exists $i \in N$ with $s_{i}=y$, there are $n-1-\delta=k$ many receivers $j \in N$ such that $s_{j}(g)=\tilde{x}(j)$. Therefore all these signals persuade exactly $k$ agents, i.e., for such $s \in S^{\pi}$ we have $\left|\left\{i \in N: \alpha_{i}^{\pi, g}\left(s_{i}(g)\right)=x\right\}\right| \geq k$ The value is equal to the empty network value, $V_{k}^{\pi}(g)=\lambda^{0}(X) \cdot 1+\lambda^{0}(Y) w_{1}=\min \left\{\frac{n+k}{k}, 1\right\}=V_{k}^{n}$.

Proof of Proposition 5.10. In the empty network the value corresponding to $k=$ $q \alpha, n=q \beta$ is the same as the value for $k^{\prime}=\alpha, n^{\prime}=\beta$, since $V_{k}^{n}\left(\lambda^{0}\right)=\min \left\{\frac{n+k}{k} \lambda^{0}(X), 1\right\}$ (Theorem 3.1) and $\frac{n+k}{k} \lambda^{0}(X)=\frac{q \alpha+q \beta}{q \beta} \lambda^{0}(X)=\frac{\alpha+\beta}{\beta} \lambda^{0}(X)$. Therefore, if the network allows uniform treatment of parts with the minimal necessary size $(q)$ so that an equal number of nodes in every part has a neighborhood with at least one $y$ message in it, the setup becomes equivalent to the empty network and allows obtaining the optimal value with private communication, so that if $V_{k}^{n}=V_{q \alpha}^{q \beta}$, then $V_{k}^{n}=V_{\alpha}^{\beta}$.

Proof of Claim 6.2. Assume the opposite, i.e. $V_{2}^{\hat{\pi}}\left(g_{2}\right)=1$. Therefore:

- $\hat{\pi}$ is optimal on $g_{2}$ and for every $t \in S^{\hat{\pi}}, t \in Z_{x}^{g_{2}}(\hat{\pi})$, so all signals in $\hat{\pi}$ implement $x$.

- for every $t \in Z_{x}^{g_{2}}(\hat{\pi})$, node 2 associates $t$ with at least one of the anchors, i.e. there exists $s \in A n(\hat{\pi})$ such that $t \in A_{2}^{\pi}(g, s)$. If $t_{2} \neq s_{2}^{\prime}$ for all $s^{\prime} \in A n(\hat{\pi})$, then agents 1,2 and 3 would vote for $y$ and the signal would not implement $x$. 
- Node 4 never receives any information from any other node, so changing anything in the other nodes' messages would not change the vote of 4 .

Observe that if player 2 is never pivotal, then any transformation of the communication strategy which preserves the other nodes' votes does not change the value of the strategy.

Claim A.2. For any $\hat{\pi} \in \Pi$ with $V_{2}^{\hat{\pi}}\left(g_{2}\right)=1$ there exists $\hat{\pi}^{\prime} \in \Pi$ such that: $(i) 2$ is never pivotal, (ii) $\left|S_{2}^{\hat{\pi}^{\prime}}\right|=1$ and (iii) $V_{2}^{\hat{\pi}^{\prime}}\left(g_{2}\right)=V_{2}^{\hat{\pi}}\left(g_{2}\right)$.

If the claim holds, then node 2 never reveals or receives any consequential information (in terms of value). That is, the situation would be equivalent to an empty network with $n=3$ and $k=2$ where $V_{2}^{\hat{\pi}^{\prime}}\left(g_{2}\right) \leq V_{2}^{3}=5 / 6<1$, which would contradict the initial assumption.

Proof of Claim A.2. (i) It is clear that for $s \in S^{\hat{\pi}}$ with $\alpha_{2}^{\hat{\pi}, g_{2}}\left(s_{2}\left(g_{2}\right)\right)=y$, node 2 is not pivotal since all signals implement $x$.

Suppose that there is a signal $t \in S^{\hat{\pi}}$ in which node 2 votes for $x$. Hence, there is at least one anchor $s \in A n(\hat{\pi})$ with $\left(s_{1}, s_{2}, s_{3}\right)=\left(t_{1}, t_{2}, t_{3}\right)$ and for every $r \in S^{\hat{\pi}}$ such that $\left(r_{1}, r_{2}, r_{3}\right)=\left(s_{1}, s_{2}, s_{3}\right)$, node 2 also votes for $x$. The possible voting patterns of nodes 1,2 and 3 in such signals are: (a) $(x, x, x) ;(b)(y, x, x) ;(c)(x, x, y) ;(d)(y, x, y)$.

In the case $(a), 2$ is not pivotal. Consider case $(b)$. It must be true that node 1 votes for $y$ because it associates $t$ with more signals than 2. In other words, in all signals $r \in S^{\hat{\pi}}$ where $\left(r_{1}, r_{2}\right)=\left(t_{1}, t_{2}\right)$ node 1 votes for $y$ and this includes the signals in which 2 does not vote for $x$. (This also includes the associated anchors.) Thus, $A_{2}^{\pi}\left(g_{2}, t\right) \subsetneq A_{1}^{\pi}\left(g_{2}, t\right)$.

Notice the trivial fact that for every $s, t \in S^{\hat{\pi}}$ with $s_{2} \neq t_{2}$ and $i \in\{1,2,3\}$, it holds that $A_{i}^{\pi}\left(g_{2}, s\right) \cap A_{i}^{\pi}\left(g_{2}, t\right)=\emptyset$, so that whenever node 2 receives a different message in different signals, these signals belong to disjoint association sets (for node 2) and the same observation holds for its neighbors, 1 and 2 .

Let $S_{2}^{\hat{\pi}}=\left\{m^{1}, \ldots, m^{\ell}\right\}$ and define the set of signals in which receiver 1 votes for $y$ and receiver 2 votes for $x$ as $T=\left\{t \in S^{\hat{\pi}} \mid \alpha_{1}^{\hat{\pi}, g_{2}}\left(t_{1}\left(g_{2}\right)\right)=y\right.$ and $\left.\alpha_{2}^{\hat{\pi}, g_{2}}\left(t_{2}\left(g_{2}\right)\right)=x\right\}$.

Define a bijection such that in signals in $\hat{\pi}$ in which 1 votes for $y$ and 2 votes for $x$, we change the message of 1 to a unique message that is specific to each distinct message of 2 and keep all other messages the same. Formally, let $T^{\prime} \subsetneq S$ and define $\phi: T \rightarrow T^{\prime}$ such that for any $t \in T$ it holds that $\phi(t)=t^{\prime}$ if $t_{1}^{\prime}=\left(t_{1}, t_{2}\right) \in S_{1}^{\prime} \backslash S_{1}^{\hat{\pi}}$ and $t_{-1}^{\prime}=t_{-1}$.

Now for any $\omega \in \Omega$ define a new strategy $\hat{\pi}^{\prime} \in \Pi$, which transforms the signals in $T$ according to $\phi$, keeps all other signals the same while preserving the probability weights:

$$
\hat{\pi}^{\prime}\left(s^{\prime} \mid \omega\right)= \begin{cases}\hat{\pi}\left(s^{\prime} \mid \omega\right) & \text { if } s^{\prime} \in S^{\hat{\pi}} \backslash T \\ \hat{\pi}\left(\phi^{-1}\left(s^{\prime}\right) \mid \omega\right) & \text { if } s^{\prime} \in T\end{cases}
$$

Let $s^{\prime} \in S^{\hat{\pi}^{\prime}}$ be such that $\phi(s)=s^{\prime}$ for some $s \in T$. Then, 


$$
\begin{aligned}
& \lambda_{1}^{s^{\prime}, g_{2}}(X)=\frac{\sum_{t^{\prime} \in A_{1}^{\hat{A}^{\prime}}\left(g_{2}, s^{\prime}\right)} \hat{\pi}^{\prime}\left(t^{\prime} \mid X\right) \lambda^{0}(X)}{\sum_{\omega \in \Omega} \sum_{t^{\prime} \in A_{1}^{\hat{\pi}^{\prime}}\left(g_{2}, s^{\prime}\right)} \hat{\pi}^{\prime}\left(t^{\prime} \mid \omega\right) \lambda^{0}(\omega)}=\frac{\sum_{t^{\prime} \in A_{1}^{\hat{A}^{\prime}}\left(g_{2}, s^{\prime}\right)} \hat{\pi}\left(\phi^{-1}\left(t^{\prime}\right) \mid X\right) \lambda^{0}(X)}{\sum_{\omega \in \Omega} \sum_{t^{\prime} \in A_{1}^{A^{\prime}}\left(g_{2}, s^{\prime}\right)} \hat{\pi}\left(\phi^{-1}\left(t^{\prime}\right) \mid \omega\right) \lambda^{0}(\omega)} \\
& =\frac{\sum_{t \in A_{1}^{\hat{\pi}}\left(g_{2}, s\right) \cap A_{2}^{\hat{\pi}}\left(g_{2}, s\right)} \hat{\pi}(t \mid X) \lambda^{0}(X)}{\sum_{\omega \in \Omega} \sum_{t \in A_{1}^{\hat{\pi}}\left(g_{2}, s\right) \cap A_{2}^{\hat{\pi}}\left(g_{2}, s\right)} \hat{\pi}(t \mid \omega) \lambda^{0}(\omega)}=\frac{\sum_{t \in A_{2}^{\hat{\pi}}\left(g_{2}, s\right) \subseteq T} \hat{\pi}(t \mid X) \lambda^{0}(X)}{\sum_{\omega \in \Omega} \sum_{t \in A_{2}^{\hat{\pi}}\left(g_{2}, s\right) \subseteq T} \hat{\pi}(t \mid \omega) \lambda^{0}(\omega)} \geq \frac{1}{2},
\end{aligned}
$$

where $\phi(t)=t^{\prime}$ and the third equality follows from the definition of $\phi ; A_{1}^{\hat{\pi}}\left(g_{2}, s\right) \cap$ $A_{2}^{\hat{\pi}}\left(g_{2}, s\right)=A_{2}^{\hat{\pi}}\left(g_{2}, s\right) \subseteq T$ follows from $A_{2}^{\pi}\left(g_{2}, t\right) \subsetneq A_{1}^{\pi}\left(g_{2}, t\right)$ and the inequality follows from the definition of case $(b)$. Similarly, it holds that $\lambda_{2}^{s^{\prime}, g_{2}}(X) \geq 1 / 2$. This implies that in $\hat{\pi}^{\prime}$ node 1 will vote for $x$ whenever 2 votes for $x$ in $\hat{\pi}^{\prime}$. Additionally, node 2 will keep its vote for $x$ in the corresponding signals in $\hat{\pi}$ and $\hat{\pi}^{\prime}$. Thus, the transformation does not change the vote of 2 in any signals. It only increases the number of $x$ votes in signals which already implement $x$ (since all signals must do under $\hat{\pi}$ ). Observe that for $s \in A_{1}^{\pi}\left(g_{2}, t\right) \backslash A_{2}^{\pi}\left(g_{2}, t\right)$ such that $t \in T$, it holds that $\alpha_{1}^{\hat{\pi}, g_{2}}\left(t_{1}\left(g_{2}\right)\right)=y$ and the transformation will not decrease the value, as in such $s$ nodes 1 and 2 must already be voting for $y$. Hence, $V_{2}^{\hat{\pi}^{\prime}}\left(g_{2}\right)=V_{2}^{\hat{\pi}}\left(g_{2}\right)$. A similar transformation can be applied in cases $(c)$ and $(d)$.

Therefore, for any communication strategy $\hat{\pi}$ with $V_{k}^{\hat{\pi}}\left(g_{2}\right)=1$, there exists $\hat{\pi}^{\prime} \in \Pi$ such that in every signal in which 2 votes for $x$ in $\hat{\pi}$, nodes 1,2 and 3 vote for $x$ in $\hat{\pi}^{\prime}$ such that $V_{2}^{\hat{\pi}^{\prime}}\left(g_{2}\right)=V_{2}^{\hat{\pi}}\left(g_{2}\right)$. Thus, 2 is never pivotal in $\hat{\pi}^{\prime}$.

(ii) Keeping the message of node 4 the same as in $\hat{\pi}$ (and in $\hat{\pi}^{\prime}$ ), from here onwards, the transformation is the same as in the star network (see proof of Proposition 5.2). Note that for two anchors $s, t \in S^{\hat{\pi}}$ with $s_{2} \neq t_{2}$, it holds that $A_{2}^{\pi}\left(g_{2}, s\right) \cap A_{2}^{\pi}\left(g_{2}, t\right)=\emptyset$. Let $S^{\prime} \subseteq S$. Define a bijection $\tau: S^{\hat{\pi}^{\prime}} \rightarrow S^{\prime}$ such that $\tau(s)=s^{\prime}$ if $s_{2}^{\prime}=x$, for $j \in\{1,3\}$, $s_{j}^{\prime}=\left(s_{j}, s_{2}\right)$, and $s_{4}^{\prime}=s_{4}$. That is, in signals in $S^{\prime}$ node 2 always observes $x$ and the messages of node 1 and 3 are modified so that they contain the information previously provided by node 2 in signal $s$. In other words, the information that node 2 reveals to node 1 and 3 is shifted to them while node 2 observes the same message in every signal.

For any $s^{\prime} \in S^{\prime}$ such that $\tau(s)=s^{\prime}$ and $\omega \in \Omega$, let $\hat{\pi}^{\prime \prime} \in \Pi$ be defined by $\hat{\pi}^{\prime \prime}\left(s^{\prime} \mid \omega\right)=$ $\hat{\pi}^{\prime}\left(\tau^{-1}\left(s^{\prime}\right) \mid \omega\right)$. As the probabilities of corresponding signals are the same under $\hat{\pi}^{\prime \prime}$ as under $\hat{\pi}^{\prime}$ and node 2's information under $\hat{\pi}^{\prime}$ is shifted to nodes 1 and 3 under $\hat{\pi}^{\prime \prime}$ (which are observed by node 2), node 2's vote does not change. Moreover, the votes of nodes 1,3 , and 4 do not change either. To see this, note that for any $i \in\{1,3,4\}$ and $t^{\prime} \in A_{i}^{\hat{\pi}^{\prime \prime}}\left(g, s^{\prime}\right)$ there exists $t \in A_{i}^{\tilde{\pi}^{\prime}}(g, s)$ such that $\tau(t)=t^{\prime}$. This, together with the definition of $\tau$ implies that $\sum_{t^{\prime} \in A_{i}^{\hat{\pi}^{\prime \prime}}\left(g, s^{\prime}\right)} \hat{\pi}^{\prime \prime}\left(t^{\prime} \mid \omega\right)=\sum_{t \in A_{i}^{\hat{\pi}^{\prime}}(g, s)} \hat{\pi}^{\prime}(t \mid \omega)$. Thus, every node has the same posterior belief upon observing $s \in S^{\hat{\pi}^{\prime}}$ and $\tau(s) \in S^{\hat{\pi}^{\prime \prime}}$.

(iii) Parts $(i)$ and (ii) imply that the value is preserved, i.e. $V_{2}^{\hat{\pi}^{\prime \prime}}\left(g_{2}\right)=V_{2}^{\hat{\pi}^{\prime}}\left(g_{2}\right)=V_{2}^{\hat{\pi}}\left(g_{2}\right)$.

Hence, for $k=2$ a communication strategy $\hat{\pi}$ with $V_{2}^{\hat{\pi}}\left(g_{2}\right)=1$ can be transformed into a strategy such that: node 2 is never pivotal, it always receives the same message and the strategy preserves the value of the initial strategy. This proves Claim A.2.

As Claim A.2 holds, we have $V_{2}^{\hat{\pi}}\left(g_{2}\right) \leq 5 / 6$, which contradicts the initial assumption that $V_{2}^{\hat{\pi}}\left(g_{2}\right)=1$. Therefore, $V_{2}^{\hat{\pi}}\left(g_{2}\right)<1$, which proves Claim 6.2. 


\section{References}

Alonso, R. and O. Câmara (2016). Persuading voters. American Economic Review $106(11), 3590-3605$.

Bardhi, A. and Y. Guo (2018). Modes of persuasion toward unanimous consent. Theoretical Economics 13(3), 1111-1149.

Bergemann, D. and S. Morris (2016). Bayes correlated equilibrium and the comparison of information structures in games. Theoretical Economics 11(2), 487-522.

Buechel, B. and L. Mechtenberg (2019). The swing voter's curse in social networks. Games and Economic Behavior 118, 241-268.

Candogan, O. (2019). Persuasion in networks: Public signals and k-cores. Working paper.

Candogan, O. and K. Drakopoulos (2020). Optimal signaling of content accuracy: Engagement vs. misinformation. Operations Research 68(2), 497-515.

Chan, J., S. Gupta, F. Li, and Y. Wang (2019). Pivotal persuasion. Journal of Economic theory 180, 178-202.

Egorov, G. and K. Sonin (2019). Persuasion on networks. Working paper.

Kamenica, E. and M. Gentzkow (2011). Bayesian persuasion. American Economic Review 101(6), 2590-2615.

Kerman, T., P. J.-J. Herings, and D. Karos (2020). Persuading strategic voters. Manuscript submitted for publication.

Kosterina, S. (2018). Persuasion with unknown beliefs. Work. Pap., Princeton Univ., Princeton, NJ.

Schnakenberg, K. E. (2015). Expert advice to a voting body. Journal of Economic Theory 160, 102-113.

Taneva, I. (2019). Information design. American Economic Journal: Microeconomics 11(4), 151-85.

Wang, Y. (2013). Bayesian persuasion with multiple receivers. Working paper. 